\title{
DERIVED FUNCTORS OF UNITARY HIGHEST WEIGHT MODULES AT REDUCTION POINTS
}

\author{
PIERLUIGI MÖSENEDER FRAJRIA
}

\begin{abstract}
The derived functors introduced by Zuckerman are applied to the unitary highest weight modules of the Hermitian symmetric pairs of classical type. The construction yields "small" unitary representations which do not have a highest weight.

The infinitesimal character parameter of the modules we consider is such that their derived functors are nontrivial in more than one degree; at the extreme degrees where the cohomology is nonvanishing, it is possible to determine the K-spectrum of the resulting representations completely. Using this information it is shown that, in most cases, the derived functor modules are unitary, irreducible, and not of highest weight type. Their infinitesimal character and lowest K-type are also easily computed.
\end{abstract}

\section{INTRODUCTION}

Cohomological parabolic induction is a general algebraic method used to construct admissible representations of a real reductive group $\mathbf{G}$ from representations of a subgroup $\mathbf{L}$ of $\mathbf{G}$. This type of induction was first introduced by Zuckerman in 1977 in unpublished lectures given at The Institute of Advanced Study. The main ingredients in the Zuckerman construction are what are now called the Zuckerman functors $\Gamma^{i}$. Using these functors he constructed representations $\operatorname{Aq}(\lambda)$ that he conjectured to be unitarizable.

Vogan [14] has proven a stronger result that implies Zuckerman's conjecture: he showed that if a unitarizable $(\mathfrak{l}, \mathbf{L} \cap \mathbf{K})$-module has infinitesimal character in an appropriate Weyl chamber, then the cohomological induced $(\mathfrak{g}, \mathbf{K})$-module is unitarizable. This result relies on a general vanishing theorem for the $\Gamma^{i}$ : there is a distinguished index $S$, the so-called middle dimension, such that, if $\mathbf{V}$ satisfies the hypothesis of Vogan's theorem, then $\Gamma^{i}(\mathbf{V})=0$ if $i \neq S$.

In this paper we construct some particular representations in a situation where the type of vanishing described above is no longer present. To describe these results we need some notation: let $\mathbf{G}^{1}$ be the universal covering group of a simple group that admits unitary highest weight modules. Let $\mathbf{K}^{1}$ be a subgroup covering a maximal compact subgroup. It is shown in [4] that to

Received by the editors February 24, 1989 and, in revised form, August 21, 1989.

1980 Mathematics Subject Classification (1985 Revision). Primary 22E46, 22 E47. 
each unitary representations of $\mathbf{K}^{1}$ we can associate a one-parameter family of unitary highest weight representation that can be described by the following diagram:

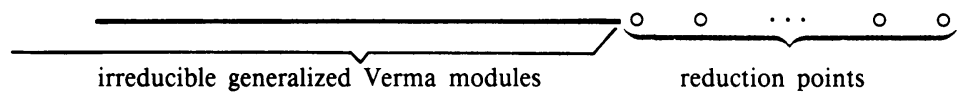

We study the Zuckerman functors at the reduction points of the family associated to the trivial representation of $\mathbf{K}^{1}$. More precisely, we analyze the case when the Lie algebras of $\mathbf{G}$ and $\mathbf{G}^{1}$ are real forms of the same complex Lie algebra and, moreover, they both correspond to Hermitian symmetric pairs of classical type. In such a situation, we show that, if $\mathbf{V}$ denotes the irreducible $\left(\mathfrak{g}, \mathbf{K}^{1}\right)$-module corresponding to a reduction point, then there is an index $i$, that depends on $\mathbf{V}$, such that $\Gamma^{i}(\mathbf{V})$ is computable. Moreover, we prove that the resulting $(\mathfrak{g}, \mathbf{K})$-module is irreducible and unitary at least for every other reduction point. In many "good" cases, however, we actually have this result for all the reduction points. The precise statements are given in $\S 7$ (Corollary 7.2). The index mentioned above is what we call the "extreme dimension", that is, the first index such that the cohomology is nonvanishing. We can also determine the $\mathbf{K}$-spectrum of $\Gamma^{i}(\mathbf{V})$ explicitly: it is mulitplicity free and the K-types form a cone in a lattice of rank less or equal to the real rank of both $\mathbf{G}$ and $\mathbf{G}^{1}$. In particular, the lowest $\mathbf{K}$-type of our representations is easily computed (Theorem 7.9).

To this moment the only known classifications of unitary representations for large rank groups are those for $\mathbf{G L}(n, K) \quad(K=\mathbb{R}, \mathbb{C}, \mathbb{H})$ [13] and for complex classical Lie groups [1]. In both cases all the unitary representations are obtained via induction or complementary series starting from basic building blocks which have small infinitesimal character and large annihilators. Our method gives a way to construct new representations with those characteristics starting from known ones (namely the unitary highest weight modules), in fact most of the modules we obtain do not have a highest weight (Corollary 7.11). This suggests that our representations could play a role in classification theories for the groups we consider similar to those cited above. They should also fit in Howe's theory of dual pairs.

There are two main steps in the proof of our results: first we give an abstract criterion for irreducibility and unitarity of the derived functors of certain representations (Theorem 5.7): we look at a highest weight module $\mathbf{M}$ that is completely reducible as a $\mathbf{K}$-module. Let $\mathscr{L}_{0}$ be the set of the irreducible representations of $\mathbf{K}$ occurring in $\mathbf{M}$ that contribute to the extreme dimension cohomology. We show that, if the infinitesimal characters of the elements of $\mathscr{L}_{0}$ are all in the same Weyl chamber and there is an element that is cyclic in $\mathscr{L}_{0}$ then the extreme dimension derived functor of $\mathbf{M}$ is irreducible and unitary.

We then give a complete description of the $k$-structure of a unitary highest weight module for the Hermitian symmetric pair $\left(\mathfrak{g}, \mathbf{K}^{1}\right)$ (Theorem 6.12). This 
allows us to apply our criterion to that case. Some case-by-case computations are needed; we give full details for type $C_{n}$ only: the other cases can be handled in a fairly similar way.

In $\S \S 2$ to 4 we give the basic results concerning Zuckerman and translation functors, and we prove a general fact about the behaviour under translation of the Shapovalov form.

In $\S 5$ we prove our abstract criterion for irreducibility and unitarity.

In $\S 6$ we establish the structural result that allows us to apply our criterion to the case of Hermitian symmetric pairs of classical type.

In $\S 7$ we carry out the complete calculations for the type $C_{n}$ case; the results for the other cases are only stated; we also give the explicit description of the K-spectrum of our representations and compute their lowest K-type.

Most of the material contained in this work is essentially the author's doctoral thesis given at Rutgers in 1988. He wishes to thank his thesis advisor, Nolan Wallach, for help and guidance. Many discussions with Dan Barbash were also very useful.

\section{Notation}

Let $G$ be the universal covering of a connected real reductive Lie group and let $K$ be a maximal connected subgroup such that $K /$ center $G$ is compact. Let $\mathfrak{g}_{0}$ and $\mathfrak{k}_{0}$ be the Lie algebras corresponding to $G$ and $K$ respectively and let $\theta$ denote the Cartan involution of $\mathfrak{g}_{0}$ giving the Cartan decomposition $\mathfrak{g}_{0}=\mathfrak{k}_{0} \oplus \mathfrak{p}_{0}$.

Let $\mathfrak{t}_{0}$ be a Cartan subalgebra (C.S.A.) of $\mathfrak{k}_{0}$ and let $\mathfrak{h}_{0}$ be the centralizer of $\mathfrak{t}_{0}$ in $\mathfrak{g}_{0} ;$ then $\mathfrak{h}_{0}$ is a C.S.A. of $\mathfrak{g}_{0}$.

We denote the complexification of a real algebra by dropping the subscript. If $\mathfrak{a} \subseteq \mathfrak{g}$ is a subalgebra, we write $\mathfrak{a}_{c}=\mathfrak{a} \cap \mathfrak{k}, \mathfrak{a}_{n}=\mathfrak{a} \cap \mathfrak{p}$; in particular, if $\mathfrak{a}$ is $\theta$-stable, we have $\mathfrak{a}=\mathfrak{a}_{c} \oplus \mathfrak{a}_{n}$.

If $E$ is a $\mathfrak{h}$-module (resp. a t-module) and $\alpha \in \mathfrak{h}^{*},\left(\alpha \in \mathfrak{t}^{*}\right)$ is a weight for $E$, let $E_{\alpha}$ denote the $\alpha$-weight space of $E$. Let $\Delta$ denote the set of roots of $\mathfrak{g}$ with respect to $\mathfrak{h}$ and let $\Delta_{\mathfrak{t}}=\Delta_{\left.\right|_{\mathfrak{t}}}$ be the set of $\mathfrak{t}$-roots for $\mathfrak{g}$. If $E \subseteq \mathfrak{g}$ is $\operatorname{ad}(\mathfrak{h})$-stable let $\Delta(E)$ be the set of roots occurring in $E$. If $E$ is ad(t)-stable we let $\Delta_{\mathfrak{t}}(E)$ denote the set of $\mathfrak{t}$-roots occurring in $E$. If there is no chance for confusion we will drop the subscript.

Let $\mathfrak{q} \subseteq \mathfrak{g}$ be a $\theta$-stable parabolic subalgebra with $\theta$-stable Levi decomposition $\mathfrak{g}=\mathfrak{m} \oplus \mathfrak{u}$. Fix $\Delta^{+} \subseteq \Delta$ a $\theta$-stable positive system compatible with q.

Let $\mathfrak{n}=\sum_{\alpha \in \Delta^{+}} \mathfrak{g}_{\alpha}, \mathfrak{b}=\mathfrak{h} \oplus \mathfrak{n}$, and set $\mathfrak{u}^{-}=\sum_{-\alpha \in \Delta(\mathfrak{u})} \mathfrak{g}_{\alpha}$; we observe that $\mathfrak{u}_{c}^{-}=\sum_{-\alpha \in \Delta\left(\mathfrak{u}_{c}\right)} \mathfrak{k}_{\alpha}$. Let $\Delta^{+}(\mathfrak{k})$ be the positive system in $\Delta(\mathfrak{k})$ corresponding to $\mathfrak{b}_{c}$.

If $E \subseteq \mathfrak{g}$ is $\operatorname{ad}(\mathfrak{h})$-stable, let $\Delta^{+}(E)=\Delta(E) \cap \Delta^{+}$and $\rho(E)=\frac{1}{2} \sum_{\alpha \in \Delta^{+}(E)} \alpha$. If $E$ is ad(t)-stable then set $\Delta_{\mathfrak{t}}^{+}(E)=\Delta_{\mathfrak{t}}(E) \cap \Delta_{l_{\mathfrak{t}}}^{+}$and $\rho_{\mathfrak{t}}(E)=\frac{1}{2} \sum_{\alpha \in \Delta_{\mathfrak{t}}^{+}(E)} \alpha$. In particular, we let $\rho$ denote $\rho(\mathfrak{g})$ and $\rho_{c}$ denote $\rho_{\mathfrak{t}}(\mathfrak{k})$. 
We fix an ad-invariant and $\theta$-invariant nondegenerate symmetric form $($, on $\mathfrak{g}$, thus $(,)_{\left.\right|_{\mathfrak{h} \times \mathfrak{h}}}$ defines an isomorphism between $\mathfrak{h}$ and $\mathfrak{h}^{*}$. If $\lambda \in \mathfrak{h}^{*}$ we let $H_{\lambda} \in \mathfrak{h}$ denote the element corresponding to $\lambda$ under this isomorphism. Under this identification $(,)_{\mathfrak{h} \times \mathfrak{h}}$ induces a nondegenerate form on $\mathfrak{h}^{*}$ that we still denote by $($,$) .$

If $\mathfrak{a}$ is a Lie algebra, let $U(\mathfrak{a})$ be its universal enveloping algebra and $Z(\mathfrak{a})$ the center of $U(\mathfrak{a})$. If $\lambda \in \mathfrak{h}^{*}$ is $\Delta^{+}(\mathfrak{m})$-dominant integral and $\mu \in \mathfrak{t}^{*}$ is $\Delta^{+}\left(\mathfrak{m}_{c}\right)$ dominant integral, we let $F(\lambda)$ and $H(\mu)$ be the irreducible finite dimensional $\mathfrak{m}$ and $\mathfrak{m}_{c}$-modules having highest weight $\lambda$ and $\mu$.

Define the generalized Verma modules

$$
\begin{aligned}
& M(\mathfrak{q}, \lambda)=U(\mathfrak{g}) \otimes_{U(\mathfrak{q})} F(\lambda), \\
& M_{c}\left(\mathfrak{q}_{c}, \mu\right)=U(\mathfrak{k}) \otimes_{U\left(\mathfrak{q}_{c}\right)} H(\mu) .
\end{aligned}
$$

Let $L(\mathfrak{q}, \lambda)$ and $L_{c}\left(\mathfrak{q}_{c}, \mu\right)$ be the irreducible quotients of $M(\mathfrak{q}, \lambda)$ and $M_{c}\left(\mathfrak{q}_{c}, \mu\right)$.

Let $X \mapsto \bar{X}$ be the conjugation of $\mathfrak{g}$ with respect to $\mathfrak{g}_{0}$. For $X \in \mathfrak{g}$ let $X^{*}=-\bar{X}$. We can extend ${ }^{*}$ to a conjugate linear antiautomorphism of $U(\mathfrak{g})$, i.e.,

$$
(X Y)^{*}=Y^{*} X^{*}, \quad X, Y \in U(\mathfrak{g})
$$

and

$$
1^{*}=1 .
$$

If $V$ is a $\mathfrak{g}$-module, a Hermitian form $($,$) on V$ is said to be $\mathfrak{g}_{0}$-invariant if

$$
(X v, w)=\left(v, X^{*} w\right) \text { for } X \in U(\mathfrak{g}), v, w \in V .
$$

Let $\mathfrak{m}_{0}=\mathfrak{m} \cap \mathfrak{g}_{0}$. We will always assume that $F(\lambda)$ admits an $\mathfrak{m}_{0}$-invariant Hermitian form $(,)_{F(\lambda)}$ or equivalently that

$$
\overline{\lambda(H)}=-\lambda(\bar{H}), \quad H \in \mathfrak{h} .
$$

If $\lambda \in \mathfrak{h}^{*}$ satisfies $(2.2)$ we say that $\lambda$ is purely imaginary.

Given $(,)_{F(\lambda)}$ one can define a $\mathfrak{g}_{0}$-invariant Hermitian form $(,)_{\lambda}$ on $M(\mathfrak{q}, \lambda)$.

The radical of this form is the maximal submodule of $M(\mathfrak{q}, \lambda)$, so $(,)_{\lambda}$ induces a form on any of its quotients and this form is nondegenerate on $L(\mathfrak{q}, \lambda)$.

Moreover, if $V$ is a quotient of $M(\mathfrak{q}, \lambda)$, then the induced form is, up to a scalar, the only $\mathfrak{g}_{0}$-invariant Hermitian form on $V$.

Let $\mathscr{W}=\mathscr{W}(\mathfrak{g}, \mathfrak{h})$ be the Weyl group of $(\mathfrak{g}, \mathfrak{h})$ and let $\mathscr{W}_{c}=\mathscr{W}(\mathfrak{k}, \mathfrak{t})$ be the Weyl group of $(\mathfrak{k}, \mathfrak{t})$.

If $\Lambda \in \mathfrak{h}^{*}$, let $\chi_{\Lambda}: Z(\mathfrak{g}) \rightarrow \mathbb{C}$ denote the infinitesimal character corresponding to $\Lambda$ via the Harish-Chandra homomorphism.

If $M$ is a $g$-module, let $M_{\chi_{\Lambda}}=\left\{v \in M \mid \exists n\right.$ such that $\left(x-\chi_{\Lambda}(x)\right)^{n} v=0$ for each $x \in Z(\mathfrak{g})\}$. 
Suppose that

$$
M=\bigoplus_{\chi_{\Lambda}} M_{\chi_{\Lambda}} .
$$

Then we write $p_{\Lambda}: M \rightarrow M_{\chi_{\Lambda}}$ for the projection corresponding to the decomposition (2.3).

If $M$ satisfies (2.3) and it is of finite length, then it is easy to see using induction on the length that there is $N \in \mathbb{N}$ such that

$$
M_{\chi_{\Lambda}}=\left\{v \in M \mid\left(x-\chi_{\Lambda}(x)\right)^{N} v=0 \quad \forall x \in Z(\mathfrak{g})\right\} .
$$

If $\mathfrak{a} \subseteq \mathfrak{b}$ are Lie algebras and $V$ is $\mathfrak{a} \mathfrak{b}$-module, we set

$$
V^{\mathfrak{a}}=\{v \in V \mid X \cdot v=0 \text { for each } X \in \mathfrak{a}\} .
$$

\section{ZUCKERMAN FUNCTORS}

If $\mathfrak{a} \supseteq \mathfrak{b}$ are Lie algebras, let $\mathscr{C}(\mathfrak{a}, \mathfrak{b})$ be the category of $\mathfrak{a}$-modules which are locally finite and completely reducible as $U(\mathfrak{b})$-modules. If $A \in \mathscr{C}\left(\mathfrak{g}, \mathfrak{m}_{c}\right)$, define $\Gamma_{A}$ to be the sum of all irreducible unitary finite-dimensional $K$-submodules of $A$.

Then

$$
\Gamma: \mathscr{C}\left(\mathfrak{g}, \mathfrak{m}_{c}\right) \rightarrow \mathscr{C}(\mathfrak{g}, \mathfrak{k})
$$

is a left exact functor. Since $\mathscr{C}\left(\mathfrak{g}, \mathfrak{m}_{c}\right)$ has enough injectives, we can form $\Gamma^{i}$ : the $i$ th right derived frunctor of $\Gamma=\Gamma^{0}$. These are the so-called Zuckerman functors.

Let $F: \mathscr{C}\left(\mathfrak{g}, \mathfrak{m}_{c}\right) \rightarrow \mathscr{C}\left(\mathfrak{k}, \mathfrak{m}_{c}\right)$ be the forgetful functor. It is shown in [3] that $F$ maps injectives to injectives, hence, for each $i, \Gamma^{i}$ and $F$ commute.

Let $S=\operatorname{dim} \mathfrak{u}_{c}$; if $w \in \mathscr{W}_{c}$ let $l(w)=\left|\left\{\alpha \in \Delta^{+}(\mathfrak{k}) \mid w(\alpha) \notin \Delta^{+}(\mathfrak{k})\right\}\right|$. The following holds:

3.1 Theorem. Let $\mu \in \mathfrak{t}^{*}$ be $\Delta^{+}(\mathfrak{k})$-dominant and purely imaginary. Let $w \in$ $\mathscr{W}_{c}$ satisfy $w \Delta^{+}(\mathfrak{k}) \supseteq \Delta^{+}\left(\mathfrak{m}_{c}\right)$. Then, for $i \in \mathbb{N}$,

$$
\Gamma^{2 S-i}\left(M_{c}\left(\mathfrak{q}_{c}, w \mu-\rho_{c}\right)\right)= \begin{cases}L_{c}\left(\mathfrak{q}_{c}, \mu-\rho_{c}\right) & \text { if } i=l(w) \text { and } \mu \text { is } \\ 0 & \text { integral and regular }, \\ \text { otherwise. }\end{cases}
$$

For a proof, see [3].

3.2 Corollary. If $\mu, w$ are as in 3.1, then

if $\mu$ is integral and regular

$$
\Gamma^{2 S-i}\left(L_{c}\left(\mathfrak{q}_{c}, w \mu-\rho_{c}\right)\right)= \begin{cases}L_{c}\left(\mathfrak{q}_{c}, \mu-\rho_{c}\right), & i=l(w), \\ 0, & i<l(w)\end{cases}
$$

otherwise

$$
\Gamma^{i}\left(L_{c}\left(\mathfrak{q}_{c}, w \mu-\rho_{c}\right)\right)=0 \quad \forall i \in \mathbb{N} .
$$


Proof. We prove the statement by induction on $S-l(w)$. If $l(w)=S$, then $(w \mu, \alpha) \leq 0$ for each $\alpha \in \Delta\left(\mathfrak{u}_{c}\right)$. This implies that $L_{c}\left(\mathfrak{q}_{c}, w \mu-\rho_{c}\right) \simeq$ $M_{c}\left(\mathfrak{q}_{c}, w \mu-\rho_{c}\right)$ (see [9]). The result then follows from 3.1.

Let $w \in \mathscr{W}_{c}$ satisfy the hypothesis and assume $i=S-l(w) \geq 1$. Assume also that the statement is true for any $w^{\prime} \in \mathscr{W}_{c}$ that satisfies the hypothesis and such that $S-l(w)<i$. Let $0=M_{0} \subset M_{1} \subset \cdots \subset M_{n}=M_{c}\left(\mathfrak{q}_{c}, w \mu-\rho_{c}\right)$ be a filtration such that

$$
M_{j} / M_{j-1} \simeq L_{c}\left(\mathfrak{q}_{c}, w_{j} \mu-\rho_{c}\right), \quad 1 \leq j \leq n,
$$

with $w_{n}=w, l\left(w_{j}\right)>l(w)$ if $j<n$, and $w_{j} \Delta^{+}(\mathfrak{k}) \supseteq \Delta^{+}\left(\mathfrak{m}_{c}\right)$ for any $j$. Such a filtration exists (this follows from [2, Théorème 7.7.7]. From the exact sequence

$$
0 \rightarrow M_{n-1} \rightarrow M_{n} \rightarrow L_{c}\left(\mathfrak{q}_{c}, w \mu-\rho_{c}\right) \rightarrow 0
$$

we obtain the exact sequence

$$
\cdots \rightarrow \Gamma^{i}\left(M_{n}\right) \rightarrow \Gamma^{j}\left(L_{c}\left(\mathfrak{q}_{c}, w \mu-\rho_{c}\right)\right) \rightarrow \Gamma^{j+1}\left(M_{n-1}\right) \rightarrow \cdots .
$$

If $\mu$ is integral and regular we see from 3.1 that

$$
\cdots \rightarrow \Gamma^{S+i}\left(M_{n}\right) \rightarrow \Gamma^{S+i}\left(L_{c}\left(\mathfrak{q}_{c}, w \mu-\rho_{c}\right)\right) \rightarrow \Gamma^{S+i+1}\left(M_{n-1}\right) \rightarrow 0
$$

is exact and that, if $r>i$,

$$
\Gamma^{S+r}\left(L_{c}\left(\mathfrak{q}_{c}, w \mu-\rho_{c}\right)\right) \simeq \Gamma^{S+r+1}\left(M_{n-1}\right) .
$$

Hence, if we prove that

$$
\Gamma^{S+r}\left(M_{n-1}\right)=0 \text { for } r \geq i,
$$

then the result follows from 3.1 .

The exact sequence

$$
0 \rightarrow M_{j-1} \rightarrow M_{j} \rightarrow L_{c}\left(\mathfrak{q}_{c}, w_{j} \mu-\rho_{c}\right) \rightarrow 0
$$

and the induction hypothesis imply that there is an onto map

$$
\Gamma^{S+r}\left(M_{j-1}\right) \rightarrow \Gamma^{S+r}\left(M_{j}\right), \quad r \geq i .
$$

Since $\Gamma^{S+r}\left(M_{0}\right)=0,(3.3)$ holds.

If $M$ is singular or nonintegral then the same argument applies.

Corollary 3.2, together with the observation preceding 3.1, are the main tools we use to compute derived functor modules.

Let $\mathfrak{a}$ be $\mathfrak{k}$ or $\mathfrak{g}$. If $A \in \mathscr{C}\left(\mathfrak{a}, \mathfrak{m}_{c}\right)$ we say that $A$ is admissible if, for each finite-dimensional $\mathfrak{m}_{c}$-module $F$,

$$
\left.\operatorname{dim} \operatorname{Hom}_{U\left(\mathfrak{m}_{c}\right)}(F, A)\right)<+\infty \text {. }
$$

Let $\mathscr{A}\left(\mathfrak{a}, \mathfrak{m}_{c}\right)$ be the category consisting of the admissible objects in $\mathscr{C}\left(\mathfrak{a}, \mathfrak{m}_{c}\right)$. If $A \in \mathscr{C}\left(\mathfrak{a}, \mathfrak{m}_{c}\right)$ (resp. $\left.A \in \mathscr{C}(\mathfrak{a}, \mathfrak{k})\right)$, let $\bar{A}^{*}$ be the dual of $A$. We set $A^{\vee}$ equal to the subspace of $\bar{A}^{*}$ consisting of the locally $U\left(\mathfrak{m}_{c}\right)$-finite (resp. $U(\mathfrak{k})$-finite) functionals. If $T: A \rightarrow B$ let $T^{\vee}: B^{\vee} \rightarrow A^{\vee}$ be the conjugate adjoint of $T$. 
3.4 Theorem. If $A \in \mathscr{A}\left(\mathfrak{a}, \mathfrak{m}_{c}\right)$ then, for each $i$, there is a natural isomorphism

$$
\text { Ө: } \Gamma^{S+i}\left(A^{\vee}\right) \rightarrow \Gamma^{S-i}(A)^{\vee} \text {. }
$$

For a proof, see [3 or 17].

If $A, B$ admit an $\mathfrak{a}_{0}$-invariant sesquilinear pairing $\psi$, then $\psi$ defines a map $\psi: A \rightarrow B^{\vee}$ by

$$
\psi^{\vee}(a)(b)=\psi(a, b) .
$$

In particular, if $A \in \mathscr{A}\left(\mathfrak{a}, \mathfrak{m}_{c}\right)$ admits an $\mathfrak{a}_{0}$-invariant Hermitian form, then $\Theta \circ \Gamma^{S+i}\left(\psi^{\vee}\right): \Gamma^{S+i}(A) \rightarrow \Gamma^{S-i}(A)^{\vee}$ defines an $\mathfrak{a}_{0}$-invariant pairing between $\Gamma^{S+i}(A)$ and $\Gamma^{S-i}(A)$. We denote this pairing by $\Gamma^{S+i}(\psi)$. If $A$ is admissible and $\psi$ is nondegenerate then $\Gamma^{S+i}(\psi)$ is nondegenerate.

This fact, together with 3.2 , implies

3.5 Corollary. If $\mu, w$ are as in 3.1, then, if $i=S-l(w)$,

$$
\Gamma^{S+i}\left(L_{c}\left(\mathfrak{q}_{c}, w \mu-\rho_{c}\right)\right) \simeq \Gamma^{S-i}\left(L_{c}\left(\mathfrak{q}_{c}, w \mu-\rho_{c}\right)\right) .
$$

Proof. 3.2 implies that $\Gamma^{S+i}\left(L_{c}\left(\mathfrak{q}_{c}, w \mu-\rho_{c}\right)\right)$ is finite dimensional. Since $\mu$ is purely imaginary, $L_{c}\left(\mathfrak{q}_{c}, w \mu-\rho_{c}\right)$ admits a $\mathfrak{k}_{0}$-invariant Hermitian form; the duality then implies the statement.

We now describe how to relate the $\mathfrak{g}$-action on $M \in \mathscr{E}\left(\mathfrak{g}, \mathfrak{m}_{c}\right)$ to the action of $\mathfrak{g}$ on $\Gamma^{i}(M)$.

Let $\mathscr{H}(K)$ be the Hecke algebra for $K$, that is the algebra of matrix entries of finite-dimensional unitary representations of $K$. Let $\mathscr{C}(K)$ be the category of locally finite unitary $K$-modules.

3.6 Lemma. If $V \in \mathscr{C}(K)$ and $M \in \mathscr{C}\left(\mathfrak{g}, \mathfrak{m}_{c}\right)$ then there exists an isomorphism

$$
T_{V}(M): \Gamma^{i}(M \otimes V) \rightarrow V \otimes \Gamma^{i}(M)
$$

which is natural in both $M$ and $V$.

Proof. In [3] it is shown that, as a k-module,

$$
\Gamma^{i}(M) \simeq H^{i}\left(\mathfrak{k}, \mathfrak{m}_{c} ; M \otimes \mathscr{H}(K)\right) .
$$

Let $T^{\prime}: V \otimes \mathscr{H}(K) \rightarrow \mathscr{H}(K) \otimes V$ be given as follows: if $f \in \mathscr{H}(K)$ and $v \in V$, let $v_{1}, \ldots, v_{d}$ be a basis of $U(\mathfrak{k}) \cdot v \subseteq V$. Let $\lambda_{1}, \ldots, \lambda_{d}$ be the dual basis and

Then

$$
\left.\left[c_{i j}(k)\right]=\lambda_{i}\left(k^{-1} v_{j}\right)\right] .
$$

$$
T^{\prime}\left(v_{j} \otimes f\right)=\sum_{r} c_{r j} f \otimes v_{r} .
$$

It is easy to check that $T^{\prime}$ is a well-defined isomorphism that intertwines $\pi \otimes L$ with $L \otimes \operatorname{trivial}(L$ is the left action of $\mathfrak{k}$ on $\mathscr{H}(K)$ ), then

$$
T_{V}(M)=H^{i}\left(\mathfrak{k}, \mathfrak{m}_{c} ; I \otimes T^{\prime}\right) .
$$

For more details, see [17]. 
We can now give the $\mathfrak{g}$-action on $\Gamma^{i}(M)$. If $M$ is a $\mathfrak{g}$-module, let

$$
m: M \otimes U(g) \rightarrow M, \quad v \otimes g \mapsto g \cdot v .
$$

We also look at $U(\mathfrak{g})$ as an object of $\mathscr{C}(K)$ under Ad.

3.9 Theorem. If $M \in \mathscr{C}\left(\mathfrak{g}, \mathfrak{m}_{c}\right)$ and $V \subseteq U(\mathfrak{g})$ is a $K$-submodule, then the following diagram is commutative:

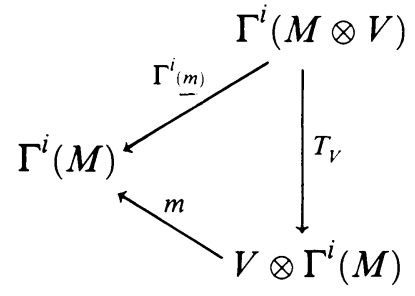

Proof. See [3 or 17].

We now want to relate the natural transformation $T_{V}=T_{V}(-)$ with the duality transformation $\boldsymbol{\theta}$.

3.10 Lemma. Suppose $F$ is a finite-dimensional object of $\mathscr{C}(K)$ and $V \in$ $\mathscr{A}\left(\mathfrak{k}, \mathfrak{m}_{c}\right)$. Then the following diagram is commutative:

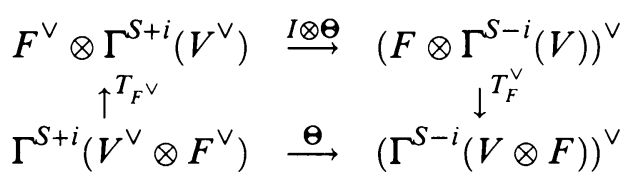

Proof. We need to recall the definition of $\Theta$. Let

$$
\left.C^{i}(V \otimes \mathscr{H}(K))=\operatorname{Hom}\left(\Lambda^{i} \mathfrak{k} / \mathfrak{m}_{c}, V \otimes \mathscr{H}(K)\right) \simeq \Lambda^{i}\left(\mathfrak{k} / \mathfrak{m}_{c}\right)^{*} \otimes V \otimes \mathscr{H}(K)\right) .
$$

We pair $\Lambda^{S+i}\left(\mathfrak{k}, / \mathfrak{m}_{c}\right)^{*}$ with $\Lambda^{S-i}\left(\mathfrak{k} / \mathfrak{m}_{c}\right)^{*}$ as follows: fix $\xi \in \Lambda^{2 S}\left(\mathfrak{k} / \mathfrak{m}_{c}\right)^{*}$, $\xi \neq 0$, and define $\langle\omega, \eta\rangle$ by $\langle\omega, \eta\rangle \xi=\omega \wedge \bar{\eta}$ for $\omega \in \Lambda^{S+i}\left(\mathfrak{k} / \mathfrak{m}_{c}\right)^{*}, \eta \in$ $\Lambda^{S-i}\left(\mathfrak{k} / \mathfrak{m}_{c}\right)^{*}$.

Next we need to define an inner product on $\mathscr{H}(K)$. We do this as follows: let $C$ be the center of $K$ and $\hat{C}$ the set of unitary characters of $C$. If $\gamma \in \hat{C}$ let

$$
\mathscr{H}(K)_{\gamma}=\{f \in \mathscr{H}(K) \mid L(c) f=\gamma(c) f \quad \forall c \in C\}
$$

Then

$$
\mathscr{H}(K)=\bigotimes_{\gamma \in \hat{C}} \mathscr{H}(K)_{\gamma} .
$$

Let $f \in \mathscr{H}(K)_{\gamma}$ and $g \in \mathscr{H}(k)_{\delta}$; we define $(,)_{2}$ by setting

$$
(f, g)_{2}= \begin{cases}0 & \text { if } \gamma \neq \delta, \\ \int_{K / C} f(k C) \overline{g(k C)} d k C & \text { if } \gamma=\delta,\end{cases}
$$

and extending $(,)_{2}$ by bilinearity. We note that the integral in (3.11) is well defined since $f \bar{g}$ is $C$-invariant and $K / C$ is compact; also, $d k C$ has been 
chosen to be normalized so that $\int_{K / C} d k C=1$. It is obvious from the definition that, if $f, g, h \in \mathscr{H}(K)$, then

$$
(f, g h)_{2}=(\bar{g} f, h)_{2} .
$$

We can now define a pairing between $C^{S+i}(V \otimes \mathscr{H}(K))$ and $C^{S-i}(V \otimes \mathscr{H}(K))$ by setting

$$
(\omega \otimes \lambda \otimes f, \eta \otimes v \otimes g)=\langle\omega, \eta\rangle \lambda(v)(f, g)_{2} .
$$

The duality transformation $\Theta$ is given by pushing down to cohomology this pairing; thus, to prove the lemma, it is enough to check that the following diagram is commutative:

$$
\begin{gathered}
\Lambda^{S+i}\left(\mathfrak{k} / \mathfrak{m}_{c}\right)^{*} \otimes V^{\vee} \otimes \mathscr{H}(K) \otimes F^{\vee} \stackrel{(,)^{\vee}}{\longrightarrow}\left(\Lambda^{S-i}\left(\mathfrak{k} / \mathfrak{m}_{c}\right)^{*} \otimes V \otimes \mathscr{H}(K) \otimes F\right)^{\vee} \\
\uparrow I \otimes T_{F}^{\prime} \vee \\
\Lambda^{S+i}\left(\mathfrak{k} / \mathfrak{m}_{c}\right)^{*} \otimes V^{\vee} \otimes F^{\vee} \otimes \mathscr{H}(K) \stackrel{(,)^{\vee}}{\longrightarrow}\left(\Lambda^{S-i}\left(\mathfrak{k} / \mathfrak{m}_{c}\right)^{*} \otimes V \otimes F \otimes \mathscr{H}(K)\right)^{\vee}
\end{gathered}
$$

That is, we must prove that

$$
\left(\omega \otimes \lambda \otimes T_{F^{\vee}}^{\prime}(\gamma \otimes f), \eta \otimes v \otimes T_{F}^{\prime}(x \otimes g)\right)=(\omega \otimes \lambda \otimes \gamma \otimes f, \eta \otimes v \otimes x \otimes g),
$$

$\omega \in \Lambda^{S+i}\left(\mathfrak{k} / \mathfrak{m}_{c}\right)^{*}, \eta \in \Lambda^{S-i}\left(\mathfrak{k} / \mathfrak{m}_{c}\right)^{*}, \lambda \in V^{\vee}, v \in V, \gamma \in F^{\vee}, x \in F$, and $f, g \in \mathscr{H}(K)$.

To do this, let $\left\{x_{1}, \ldots, x_{n}\right\}$ be a basis of $F,\left\{x^{1}, \ldots, x^{n}\right\}$ its dual basis, and take $\left\{\bar{x}^{1}, \ldots, \bar{x}^{n}\right\}$ as a basis of $F^{\vee}$ so that we can use $\left\{x_{1}, \ldots, x_{n}\right\}$ as its dual basis. Let

$$
\left[c_{i j}(k)\right]=\left[x^{i}\left(k^{-1} x_{j}\right)\right], \quad\left[\tilde{c}_{i j}(k)\right]=\left[x_{i}\left(k^{-1} \bar{x}^{j}\right)\right], \quad k \in K .
$$

Note that $\tilde{c}_{i j}(k)=\bar{c}_{j i}\left(k^{-1}\right)$ so that

$$
\sum_{r} \tilde{c}_{r i}(k) \bar{c}_{r j}(k)=\delta_{i j} .
$$

We are now ready to compute

$$
\begin{aligned}
(\omega \otimes & \left.\lambda \otimes T_{F^{\vee}}^{\prime}\left(\bar{x}^{i} \otimes f\right), \eta \otimes v \otimes T_{F}^{\prime}\left(x_{j} \otimes g\right)\right) \\
& =\left(\omega \otimes \lambda \otimes \sum_{r} \tilde{c}_{r i} f \otimes \bar{x}^{r}, \eta \otimes v \otimes \sum_{s} c_{s j} g \otimes x_{t}\right) \\
& =\langle\omega, \eta\rangle \lambda(v) \sum_{r}\left(\tilde{c}_{r i} f, c_{r j} g\right)_{2} \quad(\text { by }(3.13)) \\
& =\langle\omega, \eta\rangle \lambda(v)\left(\sum_{r} \tilde{c}_{r i} \bar{c}_{r j} f, g\right)_{2} \quad(\text { by }(3.12)) \\
& =\langle\omega, \eta\rangle \lambda(v) \delta_{i j}(f, g)_{2} \quad(\text { by }(3.14)) \\
& =\left(\omega \otimes \lambda \otimes \bar{x}^{i} \otimes f, \eta \otimes v \otimes x_{j} \otimes g\right) .
\end{aligned}
$$


3.15 Corollary. Let $F, V$ be as in 3.10. Let $\phi$ be a $\mathfrak{k}_{0}$-invariant Hermitian form on $F$ and suppose there is a $\mathfrak{k}_{0}$-invariant Hermitian form $\psi$ on $V$. Then the following diagram is commutative:

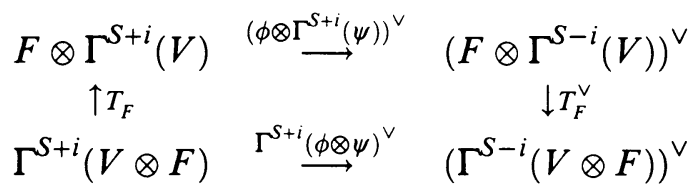

Proof. Consider the diagram expanded as follows:

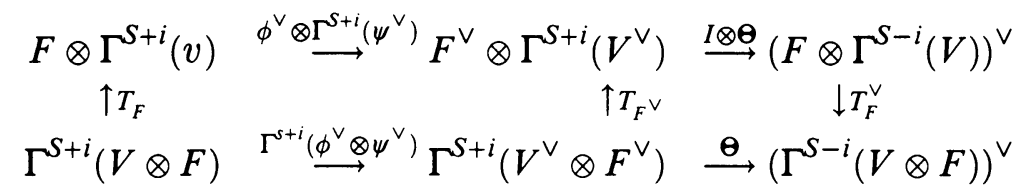

The first square is commutative by the naturality of $T_{-}$, the second by 3.10 .

\section{TRANSLATION FUnCtORS}

In this section we study the so-called translation functors and their relationwith the Zuckerman functors. We turn our attention to highest weight modules and study how invariant forms behave under translation.

If $\Lambda \in \mathfrak{h}^{*}$ let $M(\Lambda)=M(\mathfrak{b}, \Lambda)$ and $L(\Lambda)=L(\mathfrak{b}, \Lambda)$. Fix $\mu, \lambda \in \mathfrak{h}^{*}$ and $w \in \mathscr{W}$ with $\mu$ a $\Delta^{+}$-dominant integral. Let $F=L(\mu)$ (the finite-dimensional irreducible $\mathfrak{g}$-module of highest weight $\mu$ ).

Suppose $M$ is a $\mathfrak{g}$-module satisfying (2.3). We define

$$
\Psi_{\lambda}^{\lambda+w \mu}(M)=P_{\lambda+w \mu}\left(F \otimes P_{\lambda}(M)\right) .
$$

The $\Psi_{\lambda}^{\lambda+w \mu}(-)$ are the Jantzen-Zuckerman translation functors.

Let $M \in \mathscr{A}\left(\mathfrak{g}, \mathfrak{m}_{c}\right)$ be of finite length. Then $M$ satisfies (2.3) and (2.4). Using these facts and 3.9, it is easy to show that

$$
\Gamma^{i}\left(P_{\lambda}(M)\right)=P_{\lambda}\left(\Gamma^{i}(M)\right), \quad i \in \mathbb{N}, \lambda \in \mathfrak{h}^{*} .
$$

Suppose now that $\mu$ is purely imaginary and let $\phi$ be a $\mathfrak{k}_{0}$-invariant Hermitian form on $F$. We have the following.

4.1 Proposition. If $M \in \mathscr{A}\left(\mathfrak{g}, \mathfrak{m}_{c}\right)$ is of finite length then there is a natural isomorphism

$$
T_{w \mu}: \Gamma^{i}\left(\Psi_{\lambda}^{\lambda+w \mu}(M)\right) \rightarrow \Psi_{\lambda}^{\lambda+w \mu}\left(\Gamma^{i}(M)\right)
$$

such that, if there is a $\mathfrak{k}_{0}$-invariant Hermitian form $\psi$ on $M$, then the diagram

$$
\begin{array}{ll}
\Psi_{\lambda}^{\lambda+w \mu}\left(\Gamma^{S+i}(M)\right) & \stackrel{\phi^{\vee} \otimes \Gamma^{S+i}(\psi)^{\vee}}{\longrightarrow}\left(\Psi_{\lambda}^{\lambda+w \mu}\left(\Gamma^{S-i}(M)\right)\right)^{\vee} \\
\uparrow T_{w \mu} & \\
\Gamma^{S+i}\left(\Psi_{\lambda}^{\lambda+w \mu}(M)\right) & \left.\stackrel{\Gamma^{S+i}(\phi \otimes \psi}{\longrightarrow}\right)^{\vee}\left(\Gamma^{S-i}\left(\Psi_{\lambda}^{\lambda+w \mu}(M)\right)\right)^{\vee}
\end{array}
$$

is commutative. 
Proof. $T_{w \mu}$ is defined by restricting $T_{F}$ to

$$
P_{\lambda+w \mu}\left(\Gamma^{i}\left(P_{\lambda}(M) \otimes F\right)\right)=\Gamma^{i}\left(\Psi_{\lambda}^{\lambda+w \mu}(M)\right) .
$$

The second statement follows from 3.15.

For the rest of this section we assume that $\mathfrak{g}_{0}$ is a compact real form of $\mathfrak{g}$, i.e., $\theta=I$ and $\mathfrak{k}_{0}=\mathfrak{g}_{0}$.

If $\Lambda \in \mathfrak{h}^{*}$ is purely imaginary we normalize $(,)_{\Lambda}$ on $M(\Lambda)$ by setting $(1 \otimes 1,1 \otimes 1)_{\Lambda}=1$. With our assumption it also follows that $(,)_{\mu}$ is positive definite on $F$. If $w \in \mathscr{W}$ is fixed, let $P=w \Delta^{+}$and $\Gamma_{p}=\left\{\Lambda \in h^{*} \mid \Lambda\right.$ purely imaginary and $(\Lambda+\rho, \alpha)>0 \quad \forall \alpha \in P\}$.

The following is the simplest form of the translation principle (see $[10,18$ or 12]).

4.2 Theorem. If $\lambda \in \Gamma_{p}$ then

(i) $\Psi_{\Lambda+\rho}^{\Lambda+\rho+w \mu}(M(\Lambda)) \simeq M(\Lambda+w \mu)$.

(ii) $\Psi_{\Lambda+\rho}^{\Lambda+\rho+w \mu}(L(\Lambda)) \simeq L(\Lambda+w \mu)$.

From now on we let $\Psi_{w \mu}$ denote $\Psi_{\Lambda+\rho}^{\Lambda+\rho+w \mu}$. The form $\langle\rangle=,(,)_{\mu} \otimes(,)_{\Lambda}$ is clearly $\mathfrak{g}_{0}$-invariant so

$$
\langle,\rangle_{\mid \Psi_{w \mu}(M(\Lambda)) \times \Psi_{w \mu}(M(\Lambda))}=c(,)_{\Lambda+w \mu} .
$$

Our aim is to prove that $c>0$. This requires some preparation.

Let $\left\{\mu_{1}, \ldots, \mu_{d}\right\}$ be the weights of $F$ counting multiplicities and assume that they are labeled in such a way that $\mu_{i} \leq \mu_{i+1}$. Fix $\left\{f_{1}, \ldots, f_{d}\right\}$ as an orthogonal basis of $F$ so that $f_{i} \in F_{\mu_{i}}$ and let $i_{0}$ be the index such that $\mu_{i_{0}}=w \mu$.

4.3 Lemma. Let $\Lambda \in \Gamma_{P}$ and $u \in \Psi_{w \mu}(M(\Lambda))_{\Lambda+w \mu}^{\mathfrak{n}}, u \neq 0$. Then

$$
u=c_{0}\left(1 \otimes 1 \otimes f_{i_{0}}\right)+\sum_{i>i_{0}}\left(m_{i} \otimes f_{i}\right),
$$

where $m_{i} \in M(\Lambda)_{\Lambda+w \mu-\mu_{i}}$ and $c_{0} \neq 0$.

Proof. $u=\sum_{i=1}^{d}\left(m_{i} \otimes f_{i}\right)$, where $m_{i} \in M(\Lambda)_{\Lambda+w \mu-\mu_{i}}$. Let $j$ be the smallest index such that $m_{j} \neq 0$. If $X \in \mathfrak{n}$ then $X \cdot u=0$, so

$$
0=X \cdot m_{j} \otimes f_{j}+\sum_{i>j} m_{i}^{\prime} \otimes f_{i}, \quad m_{i}^{\prime} \in M(\Lambda) .
$$

Hence, $X \cdot m_{j}=0$ for each $x \in \mathfrak{n}$.

This implies that

$$
\Lambda+w \mu-\mu_{j}+\rho=t(\Lambda+\rho), \quad t \in \mathscr{W} .
$$


Now $w \mu-\mu_{j}=Q$, where $Q$ is a sum of $P$-positive roots, so

$$
t(\Lambda+\rho)=\Lambda+Q+\rho \text { or }(\Lambda+\rho)-t(\Lambda+\rho)=-Q .
$$

Since $\Lambda \in \Gamma_{P}$, this implies that $Q=0, t=1$, and $w \mu=\mu_{j}$.

For each $\alpha \in \Delta^{+}$choose $X_{\alpha} \in \mathfrak{g}_{\alpha}$ and $Y_{\alpha} \in \mathfrak{g}_{\alpha}$ such that $\left[X_{\alpha}, Y_{\alpha}\right]=H_{\alpha}$. Let $\left\{H_{i}\right\}$ be an orthogonal basis of $\mathfrak{h}$. Set

$$
D=2 \sum_{\alpha \in \Delta^{+}} Y_{\alpha} X_{\alpha}
$$

and let $C=D+2 H_{\rho}+\sum H_{i}^{2}$ be the Casimir element for $\mathfrak{g}$.

Let $\xi_{0}=w \mu, \xi_{1}, \ldots, \xi_{q}$ be the distinct weights of $F$ and let $\phi_{i}(\Lambda+\rho)=$ $\left|\Lambda+\rho+\xi_{i}\right|^{2}-|\rho|^{2}=\chi_{\Lambda+\rho+\xi_{i}}(C)$. Note that

$$
\phi_{0}(\Lambda+\rho)-\phi_{i}(\Lambda+\rho)=2\left(\Lambda+\rho, \xi_{0}-\xi_{i}\right)+\left|\xi_{0}\right|^{2}-\left|\xi_{i}\right|^{2}
$$

is a polynomial of degree one in $\Lambda+\rho$.

It is a well-known fact that the infinitesimal characters occurring in $M(\Lambda) \otimes F$ are of the type $\chi_{\Lambda+\rho+\xi_{i}}$, hence, since $\Lambda \in \Gamma_{P}$, the projection

$$
P_{\Lambda+w \mu+\rho}: M(\Lambda) \otimes F \rightarrow \psi_{w \mu}(M(\Lambda))
$$

is given by

$$
P_{\Lambda+w \mu+\rho}=\frac{\prod_{i=1}^{q}\left(c-\phi_{i}(\Lambda+\rho)\right)}{\prod_{i=1}^{q}\left(\phi_{0}(\Lambda+\rho)-\phi_{i}(\Lambda+\rho)\right)} .
$$

If $\Delta^{+}=\left\{\alpha_{1}, \ldots, \alpha_{d}\right\}$ and $I \in \mathbb{N}^{d}, I=\left(i_{1}, \ldots, i_{d}\right)$, set $Y^{I}=\prod_{j=1}^{d} Y_{\alpha_{j}}^{i_{j}}$, $|I|=\sum_{j=1}^{d} i_{j}$, and $\langle I\rangle=\sum_{j=1}^{d} i_{j} \alpha_{j}$.

\subsection{Lemma. For each $j \leq q$}

$$
\prod_{i=1}^{j}\left(C-\phi_{i}(\Lambda+\rho)\right)\left(1 \otimes 1 \otimes f_{i_{0}}\right)=\sum_{i} \sum_{\Lambda-\langle I\rangle+\mu_{i}=\Lambda+w \mu} C_{j, i, I}(\Lambda+\rho)\left(Y^{I} \otimes 1\right) \otimes f_{i}
$$

with $\operatorname{deg}\left(c_{i, I}\right)+|I| \leq j$. Moreover,

$$
c_{i_{0}, j}=\prod_{i=1}^{j}\left(\phi_{0}-\phi_{i}\right)+p
$$

with $\operatorname{deg} p<j$.

Proof. The proof is by induction on $j$. For $j=0$ the result is obvious. Assume that it is true for $j \geq 0$. 


$$
\begin{aligned}
\prod_{i=1}^{j+1}(C & \left.-\phi_{j+1}(\Lambda+\rho)\right)\left((1 \otimes 1) \otimes f_{i_{0}}\right) \\
= & \left(C-\phi_{j+1}(\Lambda+\rho)\right)\left(\prod_{i=1}^{j}\left(C-\phi_{i}(\Lambda+\rho)\right)\left((1 \otimes 1) \otimes f_{i_{0}}\right)\right) \\
= & \sum_{I, i}\left(\phi_{0}(\Lambda+\rho)-\phi_{j+1}(\Lambda+\rho)\right) c_{j, i, I}(\Lambda+\rho)\left(Y^{I} \otimes 1 \otimes f_{i}\right) \\
& +D \cdot \sum_{I, i} c_{j, i, I}(\Lambda+\rho)\left(\left(Y^{I} \otimes 1\right) \otimes f_{i}\right) .
\end{aligned}
$$

Since $\operatorname{deg}\left(\phi_{0}-\phi_{j+1}\right)=1$, the induction hypothesis implies

$$
\operatorname{deg}\left(\left(\phi_{0}-\phi_{j+1}\right) c_{j, i, I}\right)+|I| \leq j+1
$$

Furthermore, observe that

$$
X_{\alpha} \cdot\left(Y^{I} \otimes 1\right)=c_{0}(\Lambda+\rho)(1 \otimes 1)+\sum_{J} c_{J}(\Lambda+\rho)\left(Y^{J} \otimes 1\right)
$$

with $\operatorname{deg} c_{J}+|J| \leq|I|$, and that

$$
Y_{\alpha} \cdot\left(Y^{I} \otimes 1\right)=\sum_{J \neq 0} c_{J}^{\prime}(\Lambda \oplus \rho)\left(Y^{J} \otimes 1\right)
$$

with $\operatorname{deg} c_{J}^{\prime}+|J| \leq|I|+1$.

These facts together with the induction hypothesis give the first part of the statement.

Moreover,

$$
\operatorname{deg}\left(c_{j, i, I} c_{0}\right)=\operatorname{deg} c_{j, i, I}+\operatorname{deg} c_{0} \leq j
$$

SO

$$
c_{i_{0}, j+1}=\left(\phi_{0}-\phi_{j+1}\right) c_{i_{0}, j}+p^{\prime}
$$

with $\operatorname{deg} p^{\prime} \leq j$. The induction hypothesis now implies the result for $j+1$.

Set $\omega(\Lambda)=\prod_{i=1}^{q}\left(C-\phi_{i}(\Lambda+\rho)\right)\left((1 \otimes 1) \otimes f_{i_{0}}\right)$. By (3.4) we have that $\omega(\Lambda) \in \Psi_{w \mu}(M(\Lambda))_{\lambda+w \mu}$. From Theorem 3.2 we see that

$$
\Psi_{w \mu}(M(\Lambda))_{\Lambda+w \mu}=\Psi_{w \mu}(M(\Lambda))_{\Lambda+w \mu}^{\mathfrak{n}},
$$

so $\omega(\Lambda) \in \Psi_{w \mu}(M(\Lambda))_{\Lambda+w \mu}^{\mathrm{n}}$.

Let $\nu=w \rho$; then, since $\Lambda \in \Gamma_{P}$, there exists $\varepsilon>0$ such that, for each $t>-\varepsilon, \Lambda+t \nu \in \Gamma_{P}$. Fix one such $\varepsilon$ and, for each $t \in(-\varepsilon,+\infty)$, set $\omega(t)=\omega(\Lambda+t \nu), c_{i_{0}}(t)=c_{i_{0}, q}(\Lambda+t \nu+\rho)$ and, for each $t>-\varepsilon$ such that $c_{i_{0}}(t) \neq 0$, set $u(t)=\omega(t) / c_{i_{0}}(t)$.

We are about to prove the main lemma of this section. Before stating it we need to clarify some terminology. 
Suppose $A \subseteq(-\varepsilon,+\infty)$ and, for each $t \in A$, let

$$
v(t)=\sum_{i, I} a_{i, I}(t) Y^{I} \otimes 1 \otimes f_{i} \in M(\Lambda+t \nu) \otimes F .
$$

We say that $v(t)$ can be continuously extended to $(-\varepsilon,+\infty)$ if there are continuous extensions of the $c_{i, I}$ 's to $(-\varepsilon,+\infty)$.

If $t_{0} \in(-\varepsilon,+\infty)$ we define

$$
\lim _{t \rightarrow t_{0}} v(t)=\sum_{i, I} \lim _{t \rightarrow t_{0}} c_{i, I}(t)\left(\left(Y^{I} \otimes 1\right) \otimes f_{i}\right)
$$

whenever the R.H.S. makes sense.

4.6 Lemma. $u(t)$ can be extended continuously to $(-\varepsilon,+\infty)$. This extension is unique and it defines a nonzero element of $\Psi_{w \mu}(M(\Lambda+t \nu))_{\Lambda+t \nu+\rho+w \mu}^{\mathfrak{n}}$ for each $t \in(-\varepsilon,+\infty)$.

Proof. First we need to assemble some facts:

(1) $\operatorname{deg} c_{i_{0}}=q$. In particular, $c_{i_{0}}$ is not identically zero: by 4.5

$$
c_{i_{0}}(t)=\prod_{i=1}^{q}\left(\phi_{0}(\Lambda+t \nu)-\phi_{i}(\Lambda+t \nu)\right)+p(t)
$$

with $\operatorname{deg} p<q$,

$$
\begin{aligned}
& \prod_{i=1}^{q}\left(\phi_{0}(\Lambda+t \nu)-\phi_{i}(\Lambda+t \nu)\right) \\
& \quad=\prod_{i=1}^{q}\left(2\left(\Lambda+\rho+t \nu, \xi_{0}-\xi_{i}\right)+\left|\xi_{0}\right|^{2}-\left|\xi_{i}\right|^{2}\right) \\
& \quad=\prod_{i=1}^{q} 2\left(\nu, \xi_{0}-\xi_{i}\right) t^{q}+p_{1}(t) \quad \text { with } \operatorname{deg} p_{1}<q .
\end{aligned}
$$

(2) If $v=\sum_{i, I} a_{i, I}\left(Y^{I} \otimes 1\right) \otimes f_{i} \in M((\Lambda+t \nu) \otimes F$ and $X \in U(g)$ then

$$
X \cdot v=\sum_{j, J} f_{j, J}\left(a_{i, I}, t\right)\left(Y^{J} \otimes 1\right) \otimes f_{j},
$$

where the $f_{j, J}$ 's are polynomials in $a_{i, I}$ and $t$. This is obvious.

Because of (1), in order to prove the lemma, we have to show that $\lim _{t \rightarrow t_{0}} u(t)$ exists and is nonzero for each $t_{0} \in(-\varepsilon,+\infty)$.

Without loss of generality, we can assume $t_{0}=0$.

$$
\omega(t)=c_{i_{0}}(t)\left((1 \otimes 1) \otimes f_{i_{0}}\right)+\sum_{i, I \neq 0} c_{i, I}(t)\left(Y^{I} \otimes 1\right) \otimes f_{i}
$$

Let

$$
\begin{array}{rlrl}
c_{i_{0}}(t) & =t^{p_{0}} \gamma_{i_{0}}(t), & & \gamma_{i_{0}}(0) \neq 0, \\
c_{i, I}(t)=t^{P_{i, I}} \gamma_{i, I}(t), & & \gamma_{i, I}(0) \neq 0 .
\end{array}
$$


Let $M=\min \left(p_{0}, p_{i, I}\right)$. We claim that $M=p_{0}$.

Indeed, $\omega(t) \in \Psi_{w \mu}(M(\Lambda+t \nu))_{\Lambda+w \mu+t \nu}^{\mathrm{n}}$, so, for each $t \neq 0$,

(2) implies that, if $X \in U(\mathfrak{g})$,

$$
t^{-M} \omega(t) \in \Psi_{w \mu}(M(\Lambda+t \nu))_{\Lambda+w \mu+t \nu}^{\mathfrak{n}} .
$$

$$
X \cdot \lim _{t \rightarrow 0} t^{-M} \omega(t)=\lim _{t \rightarrow 0} X \cdot t^{-M} \omega(t) .
$$

Hence, $0 \neq \lim _{t \rightarrow 0} t^{-M} \omega(t) \in \Psi_{w \mu}(M(\Lambda))_{\Lambda+w \mu}^{\mathfrak{n}}$. If $M<p_{0}$

$$
\lim _{t \rightarrow 0} t^{-M} \omega(t)=\sum_{\substack{I \neq 0 \\ i \neq i_{0}}} c_{i, I}^{\prime}\left(Y^{I} \otimes 1\right) \otimes f_{i} .
$$

This is not possible because of 4.3 , so $M=p_{0}$. Set

$$
u(0)=\lim _{t \rightarrow 0} \frac{\omega(t)}{c_{i_{0}}(t)}=\lim _{t \rightarrow 0}(1 \otimes 1) \otimes f_{i_{0}}+\sum_{i, I \neq 0} t^{p_{i, I}-p_{0}} \frac{\gamma_{i, I}(t)}{\gamma_{i_{0}}(t)}\left(\left(Y^{I} \otimes 1\right) \otimes f_{i}\right) .
$$

Since $p_{i, I} \geq p_{0}$, we see that $u(0)$ is well defined and nonzero.

(2), as above, implies $u(0) \in \Psi_{w \mu}(M(\Lambda))_{\Lambda+w \mu}^{\mathfrak{n}}$.

4.7 Theorem. Let $\delta(t)=\langle u(t), u(t)\rangle$. Then $\delta(0)>0$.

Proof. (1) $\delta(t) \in \mathbb{R}$ : this is obvious.

(2) $\delta(t) \neq 0$. Suppose $\delta(t)=0.4 .2$ implies that

$$
\Psi_{w \mu}(M(\Lambda+t \nu)) \simeq M(\Lambda+t \nu+w \mu)
$$

and, by 4.6, $u(t)$ is a highest weight vector for $\Psi_{w \mu}(M(\Lambda+t \nu))$. Hence,

$$
u(t) \in\left(\Psi_{w \mu}(M(\Lambda+t \nu))\right)^{\perp},
$$

but

$$
u(t) \in \Psi_{w \mu}(M(\Lambda+t \nu))
$$

so

$$
u(t) \in\left((M(\Lambda+t \nu) \otimes F)_{\chi_{\Lambda^{\prime}}}\right)^{\perp}
$$

whenever $\chi_{\Lambda^{\prime}} \neq \chi_{\Lambda+w \mu+\rho+t \nu}$. Hence, $u(t) \in(M(\Lambda) \otimes F)^{\perp}$, but $\langle u(t),(1 \otimes 1) \otimes$ $\left.f_{i_{0}}\right\rangle=1 \neq 0$. This gives a contradiction.

(3) $\delta(0)>0$ :

$$
\delta(t)=1+\sum_{\substack{i, j \\ I+J \neq 0}} \frac{c_{i, I} \bar{c}_{j, J}}{\left|c_{i_{0}}\right|^{2}}\left(Y^{I} \otimes 1, Y^{J} \otimes 1\right)_{\Lambda+t \nu} .
$$

By the definition of $(,)_{\Lambda+t \nu}$ and 4.6, $\delta(t)$ is continuous and, by (2), nonzero for $t \geq 0$. By (1) in the proof of Lemma 4.6, $\operatorname{deg}\left|c_{i_{0}}\right|^{2}=2 q$ and, by 4.5 (and $[9$, Lemma 6]), if $I+J \neq 0$,

$$
\begin{aligned}
& \operatorname{deg} c_{i, I} \bar{c}_{j, J}\left(Y^{I} \otimes 1, Y^{J} \otimes 1\right)_{\Lambda+t \nu} \\
& \quad \leq 2 q-|I|-|J|+\min (|I|,|J|)<2 q .
\end{aligned}
$$

This implies $\lim _{t \rightarrow+\infty} \delta(t)=1$. Hence, by (2), $\delta(t)>0$ for each $t \geq 0$. 
For later use we put the statment of Theorem 4.7 in terms of diagrams:

4.8 Corollary. Suppose that $V \in \mathscr{C}(\mathfrak{g}, \mathfrak{m})$ is a quotient of $M(\Lambda)$ and $m$ : $\Psi_{w \mu}(V) \rightarrow V_{1}$ is an isomorphism. Let $\psi\left(\right.$ resp. $\left.\psi_{1}\right)$ be a $\mathfrak{g}_{0}$-invariant form on $V\left(\right.$ resp. $\left.V_{1}\right)$ which is positive definite on the highest weight space of $V$ (resp. $\left.V_{1}\right)$. Then there exists $\phi$, a positive definite form on $F$, such that the following diagram is commutative:

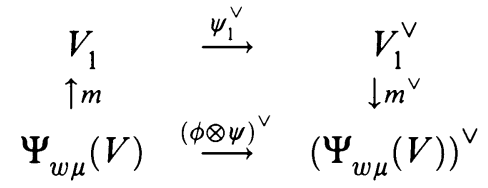

Proof. Let $\pi: M(\Lambda) \rightarrow V$ be the projection and $v(\Lambda)=(\pi \otimes \mathrm{id})(u(0))$.

$\psi_{1}(m(v(\Lambda)), m(v(\Lambda)))$ is positive so, by 4.7 , there exists $c \in \mathbb{R}, c>0$, such that $m^{\vee} \circ \psi_{1}^{\vee} \circ m=c\langle,\rangle^{\vee} \cdot \psi(\pi(1 \otimes 1), \pi(1 \otimes 1))$ is positive so there is $c_{1} \in \mathbb{R}, c_{1}>0$, such that $\psi=c_{1}(,)_{\Lambda}$; hence, $\phi=c / c_{1}(,)_{\mu}$ will do.

\section{A CRITERION FOR UNITARITY}

In this section we prove, under very strong hypotheses on $V=L(\mathfrak{q}, \Lambda)$, a criterion for the irreducibility and unitarity of $\Gamma^{i}(V)$ for a certain index $i$.

We keep the basic notation of the previous section.

Our basic assumption on $\mathfrak{q}$ is that $\left[\mathfrak{u}_{c}, \mathfrak{u}_{n}\right]=0$.

Let $\Lambda \in \mathfrak{h}^{*}$ be purely imaginary such that $(\Lambda, \alpha)=0$ for each $\alpha \in \Delta(\mathfrak{m})$. In this case $F(\Lambda)$ is one dimensional. Let $M(\Lambda)=M(\mathfrak{q}, \Lambda)$ and $L(\Lambda)=$ $L(\mathfrak{q}, \Lambda)$. If $\lambda \in \mathfrak{t}^{*}$ is $\Delta^{+}\left(\mathfrak{m}_{c}\right)$-dominant integral, then we set $M_{c}(\lambda)=M_{c}\left(\mathfrak{q}_{c}, \lambda\right)$ and $L_{c}(\lambda)=L_{c}\left(\mathfrak{q}_{c}, \lambda\right)$.

We assume

$$
\left.(,)_{\Lambda}\right|_{L(\Lambda)^{u} c} \text { is positive definite. }
$$

(5.1) implies

$$
L(\Lambda)=L(\Lambda)^{\mathfrak{u}_{c}} \oplus \mathfrak{u}_{c}^{-} L(\Lambda)
$$

If

$$
L(\Lambda)^{\mathfrak{u}_{c}}=\bigoplus_{i} H\left(\lambda_{i}\right)
$$

as an $\mathfrak{m}_{c}$-module, then (5.1) also implies

$$
L(\Lambda)=\bigoplus_{i} V_{i}
$$

with $V_{i}=U(\mathfrak{k}) \cdot H\left(\lambda_{i}\right) \simeq L_{c}\left(\lambda_{i}\right)$. Moreover, the direct sums in (5.2), (5.3), and (5.4) are orthogonal with respect to $(,)_{\Lambda}$.

Let $\pi: M(\Lambda) \rightarrow L(\Lambda)$ be the canonical projection and set $1_{\Lambda}=\pi(1 \otimes 1)$; in particular, $\pi(g \otimes 1)=g \cdot 1_{\Lambda}$.

Let $p: L(\Lambda) \rightarrow L(\Lambda)^{\mathfrak{u}_{c}}$ be the projection corresponding to the decomposition (5.2). 
Let symm: $S\left(\mathfrak{u}_{n}^{-}\right) \rightarrow U\left(\mathfrak{u}_{n}^{-}\right)$be the symmetrization map.

5.5 Lemma. As an $\mathfrak{m}_{c}$-module

$$
L(\Lambda)^{u_{c}}=p\left(\pi\left(\operatorname{symm}\left(S\left(\mathfrak{u}_{n}^{-}\right)\right) \otimes 1\right)\right) .
$$

Proof. As an $\mathfrak{m}_{c}$-module, $M(\Lambda)=U\left(\mathfrak{u}^{-}\right) \otimes 1$. So, by P.B.W., $M(\Lambda)=$ $\left(\operatorname{symm}\left(S\left(\mathfrak{u}_{n}^{-}\right)\right) \oplus \mathfrak{u}_{c}^{-} U\left(u^{-}\right)\right) \otimes 1$. Hence

$$
\begin{aligned}
& L(\Lambda)^{\mathfrak{u}_{c}}=P(\pi(M(\Lambda))) \\
& =P\left(\pi\left(\left(\operatorname{symm}\left(S\left(\mathfrak{u}_{n}^{-}\right)\right) \oplus u_{c}^{-} U\left(\mathfrak{u}^{-}\right)\right) \otimes 1\right)\right) \\
& =P\left(\pi\left(\operatorname{symm}\left(S\left(\mathfrak{u}_{n}^{-}\right)\right) \otimes 1\right)+\mathfrak{u}_{c}^{-} \pi\left(U\left(\mathfrak{u}^{-}\right) \otimes 1\right)\right) \\
& =P\left(\pi\left(\operatorname{symm}\left(S\left(u_{n}^{-}\right)\right) \otimes 1\right)\right) \quad(\text { by }(5.2)) \text {. }
\end{aligned}
$$

If $X \in S\left(\mathfrak{u}_{n}^{-}\right)$and $v \in L(\Lambda)^{\mathfrak{u}_{c}}$ set $X * v=P(\operatorname{symm}(x) \cdot v)$.

5.6 Lemma. * defines an $S\left(\mathfrak{u}_{n}^{-}\right)$-module structure on $L(\Lambda)^{\mathfrak{u}_{c}}$.

Proof. Let $X, Y \in S\left(\mathfrak{u}_{n}^{-}\right)$and $v \in L(\Lambda)^{\mathfrak{u}_{c}}$. We have to prove that $(X Y) * v=$ $X *(Y * v)$. Clearly we can assume $Y \in u_{n}^{-}$so $\operatorname{symm}(Y)=Y$. By $(5.2)$

$$
Y \cdot v=Y * v+w, \quad w \in \mathfrak{u}_{c}^{-} L(\Lambda),
$$

so $\operatorname{symm}(X) \cdot Y \cdot v=\operatorname{symm}(X)(Y * v)+\operatorname{symm}(X) w$. Since $\left[\mathfrak{u}_{n}^{-}, \mathfrak{u}_{c}^{-}\right]=0$ we have that $\operatorname{symm}(X) w \in \mathfrak{u}_{c}^{-} L(\Lambda)$, so

$$
P(\operatorname{symm}(X) \cdot Y \cdot v)=P(\operatorname{symm}(X) \cdot Y * v))=X *(Y * v) .
$$

Since $\left[\mathfrak{u}_{n}^{-}, \mathfrak{u}_{n}^{-}\right] \subseteq \mathfrak{u}_{c}^{-}$it follows that

$$
\operatorname{symm}(X) \cdot Y \equiv \operatorname{symm}(X Y) \quad \bmod \left(\mathfrak{u}_{c}^{-} U\left(\mathfrak{u}^{-}\right)\right) .
$$

Set $\mathscr{M}=\left\{\left.\lambda \in \mathfrak{t}^{*}\right|^{\lambda}\right.$ is a $\Delta^{+}\left(\mathfrak{m}_{c}\right)$-dominant integral and $H(\lambda)$ occurs in $\left.L(\Lambda)^{u_{c}}\right\}$ and $\mathscr{L}=\left\{\lambda \in \mathscr{M} \mid \lambda+\rho_{c}\right.$ is $\Delta^{+}(\mathfrak{k})$-regular and integral $\}$.

For each $\lambda \in \mathscr{L}$ let $w(\lambda)$ be the unique element of $\mathscr{W}_{c}$ such that $w(\lambda)\left(\lambda+\rho_{c}\right)$ is $\Delta^{+}(\mathfrak{k})$-dominant.

Let $l_{0}=\min \{l(w(\lambda)) \mid \lambda \in \mathscr{L}\}$ and set

$$
\mathscr{L}_{0}=\left\{\lambda \in \mathscr{L} \mid l(w(\lambda))=l_{0}\right\}
$$

Let $\mathscr{P}=S\left(U_{n}^{-}\right)^{\mathfrak{m}_{c} \cap \mathfrak{n}_{c}}$ and $T=S-l_{0}$.

5.7 Theorem. Suppose there is a $\lambda_{0} \in \mathscr{L}_{0}$ such that

(i) for each $\lambda \in \mathscr{L}_{0}, w\left(\lambda_{0}\right)\left(\lambda-\lambda_{0}\right)$ is $\Delta^{+}(\mathfrak{k})$-dominant;

(ii) if $u_{0}$ is a highest vector for $H\left(\lambda_{0}\right)$ in $L(\Lambda)^{u_{c}}$ then, for each $\lambda \in \mathscr{L}_{0}$ and $u_{\lambda} \in L(\Lambda)^{n_{c}}$, there exists $v_{\lambda} \in \mathscr{P}$ such that $v_{\lambda} * u_{0}=u_{\lambda}$.

Then $\Gamma^{S-T}(L(\Lambda))$ is irreducible and unitarizable. 
Proof. From the decomposition in (5.4) one sees that

$$
L(\Lambda)=\bigoplus_{\lambda \in \mathscr{M}} V(\lambda)
$$

where $V(\lambda)$ is isormorphic to the sum of $m(\lambda)$ copies of $L_{c}(\lambda)$.

It follows from Corollaries 3.2 and 3.5 that

$$
\Gamma^{S \pm T}(L(\Lambda))=\bigoplus_{\lambda \in \mathscr{L}_{0}} \Gamma^{S \pm T}(V(\lambda))
$$

and $\Gamma^{S \pm T}(V(\lambda))$ is a sum of $m(\lambda)$ copies of $L_{c}\left(w(\lambda)\left(\lambda+\rho_{c}\right)-\rho_{c}\right)$. We note that (ii) implies that $m\left(\lambda_{0}\right)=1$.

We claim that the k-type $\Gamma^{S \pm T}\left(V\left(\lambda_{0}\right)\right) \simeq L_{c}\left(w\left(\lambda_{0}\right)\left(\lambda_{0}+\rho_{c}\right)-\rho_{c}\right)$ is cyclic in $\Gamma^{S \pm T}(L(\Lambda))$.

To prove this, let $u_{1}, \ldots, u_{m(\lambda)}$ be an orthogonal basis of $V(\lambda)^{\mathfrak{n}_{c}}$ and let $v_{1}, \ldots, v_{m(\lambda)}$ be the elements in $\mathscr{P}$ such that $v_{i} * u_{0}=u_{i}$. Let $V_{i}=U(\mathfrak{k}) \cdot u_{i} \simeq$ $L_{c}(\lambda)$; thus,

$$
V(\lambda)=\bigoplus_{i=1, \ldots, m(\lambda)} V_{i} \text { and } \Gamma^{S \pm T}(V(\lambda))=\bigoplus_{i=1, \ldots, m(\lambda)} \Gamma^{S \pm T}\left(V_{i}\right) .
$$

Since $v_{i} \in \mathscr{P}$ and $\left[\mathfrak{u}_{n}^{-}, \mathfrak{u}_{c}^{-}\right]=0, \operatorname{symm}\left(v_{i}\right)$ is an extremal vector for a finite-dimensional irreducible $\mathfrak{k}$-submodule $F_{i}$ of $U(\mathfrak{g})$. (Here the action is ad.) From (i) it follows that the highest weight of $F_{i}$ is $\mu=w\left(\lambda_{0}\right)\left(\lambda-\lambda_{0}\right)$. Let $w=w\left(\lambda_{0}\right)^{-1}$ and let $m: F_{i} \otimes V\left(\lambda_{0}\right) \rightarrow L(\Lambda)$ be the multiplication map (see (3.8)).

Since $\lambda_{0}+\rho_{c}$ is regular and $V\left(\lambda_{0}\right) \simeq L_{c}\left(\lambda_{0}\right)$, it follows from (i), as in the discussion after Lemma 4.5 , that

$$
P_{\lambda+\rho_{c}}\left(\operatorname{symm}\left(v_{i}\right) \otimes u_{0}\right) \in\left(P_{\lambda+\rho_{c}}\left(F_{i} \otimes V\left(\lambda_{0}\right)\right)\right)^{\mathfrak{n}_{c}}=\left(\Psi_{w \mu}\left(V\left(\lambda_{0}\right)\right)\right)^{\mathrm{n}_{c}} .
$$

Since the action of $Z(\mathfrak{k})$ preserves the decomposition (5.2), it follows that $P \circ P_{\lambda+\rho_{c}}=P_{\lambda+\rho_{c}} \circ P$ on $L(\Lambda)$, so

$$
\begin{aligned}
m \circ P_{\lambda+\rho}\left(\operatorname{symm}\left(v_{i}\right) \otimes u_{0}\right) & =P_{\lambda+\rho_{c}}\left(\operatorname{symm}\left(v_{i}\right) \cdot u_{0}\right) \\
& =P\left(P_{\lambda+\rho_{c}}\left(\operatorname{symm}\left(v_{i}\right) \cdot u_{0}\right)\right) \\
& =P_{\lambda+\rho_{c}}\left(P\left(\operatorname{symm}\left(v_{i}\right) \cdot u_{0}\right)\right) \\
& =P_{\lambda+\rho_{c}}\left(v_{i} * u_{0}\right)=u_{i} .
\end{aligned}
$$

The second equality follows from (5.8). In light of 4.2 this implies that $m$ defines an isomorphism

$$
m: \Psi_{w \mu}\left(V\left(\lambda_{0}\right)\right) \rightarrow V_{i}
$$

so

$$
\Gamma^{S \pm T}(m): \Gamma^{S \pm T}\left(\Psi_{w \mu}\left(V\left(\lambda_{0}\right)\right)\right) \rightarrow \Gamma^{S \pm T}\left(V_{i}\right)
$$

are isomorphisms. 
Let $T_{w \mu}$ be as in 4.1. Then, by 3.9,

$$
m=\Gamma^{S \pm T}(m) \circ T_{w \mu}^{-1}: \Psi_{w \mu}\left(\Gamma^{S \pm T}\left(V\left(\lambda_{0}\right)\right)\right) \rightarrow \Gamma^{S \pm T}\left(V_{i}\right)
$$

are both isomorphisms. This proves our assertion.

Suppose now that $M \subseteq \Gamma^{S-T}(L(\Lambda))$ is a proper submodule. Then, by cyclicity, $\Gamma^{S-T}\left(V\left(\lambda_{0}\right)\right) \cap M=\{0\}$. Since $\Gamma^{S+T}\left((,)_{\Lambda}\right)$ is a nondegenerate $\mathfrak{g}_{0}$ invariant pairing between $\Gamma^{S-T}(L(\Lambda))$ and $\Gamma^{S+T}(L(\Lambda))$, and since $\Gamma^{S-T}\left(V\left(\lambda_{0}\right)\right)$ occurs with multiplicity one, $\Gamma^{S+T}\left(V\left(\lambda_{0}\right)\right) \subseteq M^{\perp} \subseteq \Gamma^{S+T}(L(\Lambda))$. Therefore $M^{\perp}=\Gamma^{S+T}(L(\Lambda))$. Hence $M=\{0\}$.

We have proven that $\Gamma^{S-T}(L(\Lambda))$ is irreducible. Obviously, the same proof gives the irreducibility of $\Gamma^{S+T}(L(\Lambda))$.

Since $m\left(\lambda_{0}\right)=1, U(\mathfrak{g})^{\mathfrak{k}}$, the centralizer of $\mathfrak{k}$ in $U(\mathfrak{g})$, acts on $V\left(\lambda_{0}\right)$ by a scalar.

Theorem 3.9 implies that $U(\mathfrak{g})^{\mathfrak{k}}$ acts on $\Gamma^{S+T}\left(V\left(\lambda_{0}\right)\right)$ and $\Gamma^{S-T}\left(V\left(\lambda_{0}\right)\right)$ by the same scalar (see [3]). Hence $\Gamma^{S+T}\left(V\left(\lambda_{0}\right)\right)$ and $\Gamma^{S-T}\left(V\left(\lambda_{0}\right)\right)$ are isomorphic as $U(\mathfrak{g})^{\mathfrak{k}}$-modules.

Corollary 3.5 says that $\Gamma^{S+T}(L(\Lambda))$ and $\Gamma^{S-T}(L(\Lambda))$ are isomorphic as $\mathfrak{k}$ modules, hence, a well-known result of Harish-Chandra [5] implies that there is a $\mathfrak{g}$-isomorphism

$$
\mathscr{G}: \Gamma^{S-T}(L(\Lambda)) \rightarrow \Gamma^{S+T}(L(\Lambda))
$$

The map

$$
\Gamma^{S+T}\left((,)_{\Lambda}\right)^{\vee} \circ \mathscr{G}: \Gamma^{S-T}(L(\Lambda)) \rightarrow\left(\Gamma^{S-T}(L(\Lambda))\right)^{\vee}
$$

defines a nondegenerate $\mathfrak{g}_{0}$-invariant sesquilinear form on $\Gamma^{S-T}(L(\Lambda))$ that we denote by $(,)_{\Gamma}$.

We can choose $\mathscr{G}$ so that $(,)_{\Gamma}$ is Hermitian. This is true, for example, because $m\left(\lambda_{0}\right)=1$ and $\Gamma^{S-T}(L(\Lambda))$ is irreducible.

Recall that our basis of $V(\lambda)^{\mathrm{n}} c$ is orthogonal with respect to $(,)_{\Lambda}$. It is apparent from the explicit formula defining $\Gamma^{S+T}\left((,)_{\Lambda}\right)$ (see the proof of I.2.10) that, if $i \neq j$,

$$
\left.\Gamma^{S+T}\left((,)_{\Lambda}\right)\right|_{\Gamma^{S+T}\left(V_{i}\right) \times \Gamma^{S-T}\left(V_{j}\right)}=0 .
$$

We observe that

$$
\mathscr{G}\left(\Gamma^{S-T}\left(V_{i}\right)\right) \subseteq \Gamma^{S+T}\left(V_{i}\right) .
$$

In fact, since $\mathscr{G}$ is a $\mathfrak{g}$-map, the following diagram is commutative:

$$
\begin{array}{ccc}
\Gamma^{S-T}\left(V_{i}\right) & \stackrel{\mathscr{G}}{\longrightarrow} & \Gamma^{S+T}(L(\Lambda)) \\
\underset{\uparrow m}{\uparrow m} & & \stackrel{\uparrow m}{\longrightarrow} \\
\Psi_{w \mu}\left(\Gamma^{S-T}\left(V\left(\lambda_{0}\right)\right)\right) & \stackrel{I \otimes \mathscr{G}}{\longrightarrow} & \Psi_{w \mu}\left(\Gamma^{S+T}\left(V\left(\lambda_{0}\right)\right)\right)
\end{array}
$$


Hence,

$$
\begin{aligned}
\mathscr{G}\left(\Gamma^{S-T}\left(V_{i}\right)\right) & =m \circ(I \otimes \mathscr{G})\left(\Psi_{w \mu}\left(\Gamma^{S-T}\left(V\left(\lambda_{0}\right)\right)\right)\right) \\
& =m\left(\Psi_{w \mu}\left(\Gamma^{S+T}\left(V\left(\lambda_{0}\right)\right)\right)\right)=\Gamma^{S+T}\left(V_{i}\right) .
\end{aligned}
$$

It follows that the decomposition

$$
\Gamma^{S-T}(V(\lambda))=\bigoplus_{i=1, \ldots, m(\lambda)} \Gamma^{S-T}\left(V_{i}\right)
$$

is orthogonal with respect to $(,)_{\Gamma}$.

We now want to compute the signature of $(,)_{\Gamma}:\left.(,)_{\Gamma}\right|_{L(\Lambda)^{n_{c}}}$ is positive definite so Corollary 4.8 implies that there is a positive definite form $\phi_{i}$ on $F_{i}$ such that the following diagram is commutative:

$$
\begin{array}{ccc}
V_{i} & \stackrel{(,)_{a}^{\vee}}{\longrightarrow} & V_{i}^{\vee} \\
\uparrow m & & \downarrow m^{\vee} \\
\Psi_{w \mu}\left(V\left(\lambda_{0}\right)\right) & \stackrel{\left(\phi_{i} \otimes(,)\right)^{\vee}}{\longrightarrow} & \left(\Psi_{w \mu}\left(V\left(\lambda_{0}\right)\right)\right)^{\vee}
\end{array}
$$

Hence, the rear top square of the following prism is commutative:

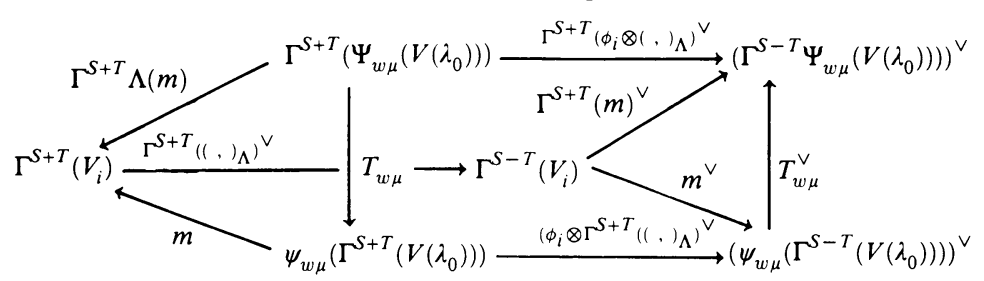

The sides and the front are also commutative because of 3.9 and 4.1 , thus the bottom square is also commutative. Thus the commutative diagram (5.9) implies that

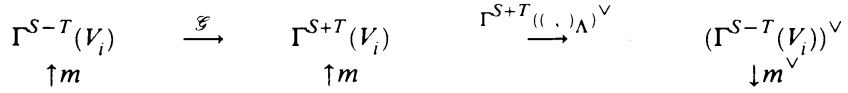

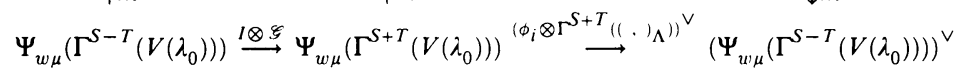

is commutative. That is,

is commutative.

$$
\begin{aligned}
& \Gamma_{\uparrow m}^{S-T}\left(V_{i}\right) \\
& \stackrel{(,)_{\Gamma}^{\vee}}{\longrightarrow}\left(\Gamma^{S-T}\left(V_{i}\right)\right)^{\vee} \\
& \downarrow \mathrm{m}^{\mathrm{v}} \\
& \Psi_{w \mu}\left(\Gamma^{S-T}\left(V\left(\lambda_{0}\right)\right)\right) \stackrel{\left(\phi_{i} \otimes(,)_{\Gamma}\right)^{\vee}}{\longrightarrow}\left(\Psi_{w \mu}\left(\Gamma^{S-T}\left(V\left(\lambda_{0}\right)\right)\right)\right)^{\vee}
\end{aligned}
$$

Since $\phi_{i}$ is positive definite, $\left.(,)_{\Gamma}\right|_{\Gamma^{S-T}\left(V_{i}\right) \times \Gamma^{S-T}\left(V_{i}\right)}$ has the same sign as $\left.(,)_{\Gamma}\right|_{\Gamma^{S-T}\left(V\left(\lambda_{0}\right)\right) \times \Gamma^{S-T}\left(V\left(\lambda_{0}\right)\right)} \cdot$

Hence, $(,)_{\Gamma}$ is definite. Therefore $\Gamma^{S-T}(L(\Lambda))$ is unitarizable.

\section{HeRmitian SYMMETRIC PAIRS}

In this section we prove some structural lemmas that allow us to describe the structure of $L(\Lambda)^{\mathfrak{u}}{ }^{c}$ as $\mathfrak{m}_{c}$-module in the case where both $\mathfrak{p}$ and $\mathfrak{u}$ are abelian; that is, when both $(\mathfrak{g}, \mathfrak{k})$ and $(\mathfrak{g}, \mathfrak{m})$ are Hermitian symmetric pairs. 
Let $\mathfrak{g}$ be a complex reductive Lie algebra such that $[\mathfrak{g}, \mathfrak{g}]$ is simple. Fix $\mathfrak{g}_{u}$ as a compact real form of $\mathfrak{g}$. Let $\mathfrak{t}_{0}$ be a maximal abelian subalgebra of $\mathfrak{g}_{u}$ and set $\mathfrak{t}=\left(\mathfrak{t}_{0}\right)_{\mathbb{C}}$.

Fix $H_{0}, H_{1} \in i t_{0}$ linearly independent vectors such that the eigenvalues of $\operatorname{ad}\left(H_{i}\right)$ are contained in $\{-1,0,1\}$.

Let

$$
\Theta=\exp \left(\pi i \operatorname{ad}\left(H_{0}\right)\right), \quad \Theta_{1}=\exp \left(\pi i \operatorname{ad}\left(H_{1}\right)\right) .
$$

Let $\tau$ be the conjugation in $\mathfrak{g}$ with respect to $\mathfrak{g}_{u}$ and set

$$
\mathfrak{g}_{0}=\{X \in \mathfrak{g} \mid X=\Theta \circ \tau(X)\}, \quad \mathfrak{g}_{0}^{1}=\left\{X \in \mathfrak{g} \mid X=\Theta_{1} \circ \tau(X)\right\} .
$$

We observe that $\left(\mathfrak{g}_{0}^{1}\right)_{\mathbb{C}}=\left(\mathfrak{g}_{0}\right)_{\mathbb{C}}=\mathfrak{g} . \Theta$ and $\Theta_{1}$ define Cartan decompositions on $\mathfrak{g}_{0}$ and $\mathfrak{g}_{0}^{1}$ :

$$
\mathfrak{g}_{0}=\mathfrak{k}_{0} \oplus \mathfrak{p}_{0}, \quad \mathfrak{g}_{0}^{1}=\mathfrak{k}_{0}^{1} \oplus \mathfrak{p}_{0}^{1} .
$$

Let $\mathfrak{g}=\mathfrak{m} \oplus \mathfrak{u}$ be the parabolic subalgebra associated to $H_{1}$, i.e.,

$$
\Delta(\mathfrak{m})=\left\{\beta \in \Delta(\mathfrak{g}) \mid \beta\left(H_{1}\right)=0\right\}, \quad \Delta(\mathfrak{u})=\left\{\beta \in \Delta(\mathfrak{g}) \mid \beta\left(H_{1}\right)=1\right\} .
$$

The definitions imply that $\left(\mathfrak{k}_{0}^{1}\right)_{\mathbb{C}}=\mathfrak{m}$ and $\left(\mathfrak{p}_{0}^{1}\right)_{\mathbb{C}}=\mathfrak{u}^{-} \oplus \mathfrak{u}$. It is also clear that $\mathfrak{q}$ and $\mathfrak{m}$ are $\Theta$-stable and that $\mathfrak{u}$ is abelian.

If $\mathfrak{d}$ is a complex reductive Lie algebra we let $\operatorname{rank}_{\mathbb{C}}(\mathfrak{d})$ denote the rank of $\mathfrak{d}$. If $\mathfrak{d}_{0}$ is a real reductive Lie algebra, let $\operatorname{rank}_{\mathbb{R}}\left(\mathfrak{d}_{0}\right)$ denote the real rank of $\mathfrak{d}_{0}$. If $r=\operatorname{rank}_{\mathbb{R}}\left(\mathfrak{g}_{0}^{1}\right)$, then to each positive system $\Delta^{+} \subset \Delta$ compatible with $\mathfrak{q}$ is associated an ordered set $\gamma_{1} \leq \cdots \leq \gamma_{r}$ of strongly orthogonal roots in $\Delta(\mathfrak{u})$ having the following properties:

(1) $2 \frac{\left(\alpha, \gamma_{i}\right)}{\left(\gamma_{i}, \gamma_{i}\right)} \in\{0,1,2\}$ for each $\alpha \in \Delta(\mathfrak{u})$ and, if $2 \frac{\left(\alpha, \gamma_{i}\right)}{\left.\gamma_{i}, \gamma_{i}\right)}=2$, then $\alpha=\gamma_{i}$.

(2) $2 \frac{\left(\alpha, \gamma_{i}\right)}{\left(\gamma_{i}, \gamma_{i}\right)} \in\{-1,0,1\}$ for each $\alpha \in \Delta(\mathfrak{m})$. Moreover, if $\alpha \in \Delta^{+}(\mathfrak{m})$ and $2 \frac{\left(\alpha, \gamma_{i}\right)}{\left(\gamma_{i}, \gamma_{i}\right)}=1$, then there is $j<i$ such that $2 \frac{\left(\alpha, \gamma_{i}\right)}{\left(\gamma_{i}, \gamma_{i}\right)}=-1$

(see $[7,8$, or 15$])$.

6.2 Lemma. Let $s \leq r$ and $\left\{\gamma_{1}, \ldots, \gamma_{s}\right\} \subseteq \Delta(\mathfrak{u})$ be a set of strongly orthogonal roots satisfying property $(6.1(1))$. Then there is a positive system $\Delta^{+} \subset \Delta$ compatible with $\mathfrak{q}$ such that, if $\left\{\eta_{1}, \ldots, \eta_{r}\right\}$ are the strongly orthogonal roots associated with $\Delta^{+}$, then $\gamma_{i}=\eta_{i}, 1 \leq i \leq s$.

Proof. Set $h_{i}=H_{\gamma_{i}} /\left(\gamma_{i}, \gamma_{i}\right)$ and $H_{i}=H_{1}-\sum_{j=1}^{i-1} h_{j}, 1 \leq i \leq s+1$. Suppose that $\sum c_{i} h_{i}=0$; then

$$
\left(\sum c_{i}\right) H_{1}=\sum_{i>1} c_{i}\left(\sum_{j=1}^{i-1} h_{j}\right) .
$$


Thus, by the linear independence of the $h_{i}$ 's, we have that $\sum c_{i}=0$ implies that $c_{i}=0$ for each $i$. If $\sum c_{i} \neq 0$ then we can write $H_{1}=\sum c_{i}^{\prime} h_{i}$. It follows that $1=\gamma_{i}\left(H_{1}\right)=c_{i}^{\prime}$; hence, $H_{1}=\sum h_{i}$.

Set

$$
B^{-}= \begin{cases}\left\{H_{i}\right\}_{1 \leq i \leq s} & \text { if } H_{1}=\sum h_{i}, \\ \left\{H_{i}\right\}_{1 \leq i \leq s+1} & \text { otherwise. }\end{cases}
$$

The discussion above shows that $B^{-}$is an ordered basis of $\mathfrak{t}^{-}=\operatorname{span}\left(\left\{H_{i}\right\}\right)$ $\subset i \mathfrak{t}_{0}$. Let $\mathfrak{t}^{+}=\left(\mathfrak{t}^{-}\right)^{\perp} \subset i \mathfrak{t}_{0}$ and let $B^{+}$be any ordered basis of $\mathfrak{t}^{+}$. If we set $H^{+} \geq H^{-}$for each $H^{+} \in B^{+}$, and $H^{-} \in B^{-}$, then $B=B^{+} \cup B^{-}$is an ordered basis of $i \mathrm{t}_{0}$. We order $\left(i \mathrm{t}_{0}\right)^{*}$ lexicographically according to the choice of $B$ and let $\Delta^{+}$be the corresponding set of positive roots in $\Delta$.

Since $\alpha\left(H_{1}\right)=1$ for each $\alpha \in \Delta(\mathfrak{u})$, it follows that $\Delta(\mathfrak{u}) \subset \Delta^{+}$. Recall that the strongly orthogonal roots $\left\{\eta_{1}, \ldots, \eta_{r}\right\}$ are constructed inductively as follows: $\eta_{1}$ is the lowest element of $\Delta(\mathfrak{u})$ and $\eta_{i}$ is the lowest element of

$$
\left\{\alpha \in \Delta(\mathfrak{u}) \mid\left(\alpha, \eta_{j}\right)=0, j<i\right\} .
$$

By property (6.1(1)), if $\alpha \in \Delta(\mathfrak{u})$, then $\alpha\left(H_{1}\right)=1$ and $\alpha\left(H_{2}\right) \geq 0$. Moreover, $\alpha\left(H_{2}\right)=0$ if and only if $\alpha=\gamma_{1}$, thus $\gamma_{1}=\eta_{1}$.

If $i>1$ assume by induction that $\gamma_{j}=\eta_{j}$ for $j<i$. Suppose first that $H_{1} \neq \sum h_{i}$ or, if $H_{1}=\sum h_{i}$, that $i<s$. If $\beta \in\left\{\alpha \in \Delta(\mathfrak{u}) \mid\left(\alpha, \gamma_{j}\right)=0\right.$, $j<i\}$ then, by property $(6.1(1)), \beta\left(H_{j}\right)=1$ for each $j \leq i$ and $\beta\left(H_{i+1}\right) \geq 0$. Moreover, $\beta\left(H_{i+1}\right)=0$ if and only if $\beta=\gamma_{i}$, hence $\gamma_{i}=\eta_{i}$.

If $i=s$ and $H_{1}=\sum h_{i}$, then $\beta \in\left\{\alpha \in \Delta(\mathfrak{u}) \mid\left(\alpha, \gamma_{j}\right)=0, j<s\right\}$ if and only if $\beta\left(H_{1}\right)=\beta\left(h_{s}\right)=1$; therefore, by (6.1(1)), $\beta=\gamma_{s}$ and $\gamma_{s}=\eta_{s}$.

The next result is due to Schmid [11].

6.3 Proposition. Suppose that a positive system $\Delta^{+} \subset \Delta$ compatible with $\mathfrak{q}$ has been fixed and let $\left\{\gamma_{i}\right\}_{i=1, \ldots, r}$ be the corresponding set of strongly orthogonal roots. Then the highest weights of $S\left(\mathfrak{u}^{-}\right)$as an $\mathfrak{m}$-module are precisely those of the form $-\sum m_{i} \gamma_{i}, m_{1} \geq \cdots \geq m_{r} \geq 0, m_{i} \in \mathbb{N}$, each occurring with multiplicity one.

Let $v_{i} \in S\left(\mathfrak{u}^{-}\right)^{\mathrm{m} \cap \mathfrak{n}}$ be an element of weight $\mu_{i}=\sum_{j=1}^{i} \gamma_{i} \cdot 6.3$ implies that

$$
S\left(\mathfrak{u}^{-}\right)^{\mathfrak{m} \cap \mathfrak{n}}=\mathbb{C}\left[v_{1}, \ldots, v_{r}\right] .
$$

In [15], for each $i=1, \ldots, r$ a subalgebra $\mathfrak{g}_{i} \subseteq \mathfrak{g}$ is constructed having the property that

$$
v_{j} \in S\left(\mathfrak{u}^{-} \cap \mathfrak{g}_{i}\right), \quad j \leq i,
$$

and

$$
\operatorname{rank}_{\mathbb{R}}\left(\mathfrak{g}_{i} \cap \mathfrak{g}_{0}^{1}\right)=i .
$$

We remark that both $v_{i}$ and $\mathfrak{g}_{i}$ depend on the choice of $\Delta^{+}$.

Let $\tilde{\Lambda}$ be the element of $t^{*}$ corresponding to $H_{1}$ via $($,$) . Let \eta$ be a long root in $\Delta$ and set $\Lambda_{1}=\frac{1}{2}(\eta, \eta) \tilde{\Lambda}$. The following result was first proved in [15]. 
6.6 Theorem. There is a constant $C=C\left(\mathfrak{g}_{0}^{1}\right)$, depending only on $\mathfrak{g}_{0}^{1}$, such that $L\left(-z \Lambda_{1}\right)$ is unitarizable with respect to $\mathfrak{g}_{0}^{1}$ if and only if $z=C R, R=$ $0,1, \ldots, r-1$, or $z>C(r-1), z \in \mathbb{R}$. Also,

$$
L\left(-z \Lambda_{1}\right) \cong M\left(-z \Lambda_{1}\right) \text { if } z>C(R-1) .
$$

If $\Lambda=-C R \Lambda_{1}, R=0,1, \ldots, r-1$, then $v_{i} \cdot 1_{\Lambda}=0$ in $L(\Lambda)$ for $i>R$.

We will be studying the Zuckerman functors of $L(\Lambda)$ for $\Lambda=-C R \Lambda_{1}$, $R=0,1, \ldots, r-1$.

Let $\langle,\rangle_{\Lambda}$ be the $\mathfrak{g}_{0}^{1}$-invariant inner product on $L(\Lambda)$. It is shown in [5] that the positivity of $\langle,\rangle_{\Lambda}$ implies that $L(\Lambda)$ satisfies condition (5.1). In order to implement Theorem 5.7 we need to know the structure of $L(\Lambda)^{\mathfrak{u}_{c}}$ as an $\mathfrak{m}_{c}$-module. The study of such a structure requires some preparation.

Let $\mathfrak{l}_{0}=\mathfrak{g}_{0} \cap \mathfrak{g}_{0}^{1}$ and $\mathfrak{l}=\left(\mathfrak{l}_{0}\right)_{\mathbb{C}} \cdot \Theta_{\left.\right|_{\mathfrak{t}_{0}}}$ defines a Cartan involution on $\mathfrak{l}_{0}$ giving the decomposition

$$
\mathfrak{l}_{0}=\mathfrak{k}\left(\mathfrak{l}_{0}\right) \oplus \mathfrak{p}\left(\mathfrak{l}_{0}\right)
$$

One checks that

$$
\left(\mathfrak{k}\left(\mathfrak{l}_{0}\right)\right)_{\mathbb{C}}=\mathfrak{m}_{c} \text { and }\left(\mathfrak{p}\left(\mathfrak{l}_{0}\right)\right)_{\mathbb{C}}=\mathfrak{u}_{n}^{-} \oplus \mathfrak{u}_{n}
$$

In analogy with $\mathfrak{g}_{0}^{1}$, we have that $\mathfrak{p}=\mathfrak{p}^{+} \oplus \mathfrak{p}^{-}$, where $\Delta\left(\mathfrak{p}^{ \pm}\right)=\{\beta \in$ $\left.\Delta(\mathfrak{g}) \mid \beta\left(H_{0}\right)= \pm 1\right\}, \quad \Delta(\mathfrak{k})=\left\{\beta \in \Delta(\mathfrak{g}) \mid \beta\left(H_{0}\right)=0\right\}$.

Let $\mathfrak{u}_{n 1}=\mathfrak{u} \cap \mathfrak{p}^{+}$and $\mathfrak{u}_{n 2}=\mathfrak{u} \cap \mathfrak{p}^{-}$.

Since $\mathfrak{m}_{c}=\mathfrak{k} \cap \mathfrak{m}$, it is obvious that $\left[\mathfrak{m}_{c}, \mathfrak{k}\right] \subset \mathfrak{k},\left[\mathfrak{m}_{c}, \mathfrak{p}^{+}\right] \subset \mathfrak{p}^{+},\left[\mathfrak{m}_{c}, \mathfrak{p}^{-}\right] \subset$ $\mathfrak{p}^{-}$, and $\left[\mathfrak{m}_{c}, \mathfrak{u}\right] \subset \mathfrak{u}$. This implies that as an $\mathfrak{m}_{c}$-module,

$$
\mathfrak{u}=\mathfrak{u}_{n 1} \oplus \mathfrak{u}_{c} \oplus \mathfrak{u}_{n 2} .
$$

From now on we fix a positive system $\Delta^{+}\left(\mathfrak{m}_{c}\right) \subset \Delta\left(\mathfrak{m}_{c}\right)$. Let $n=\operatorname{rank}_{\mathbb{C}}(\mathfrak{g})$ and $\left\{t_{i}\right\}_{i=1, \ldots, n}$ be a basis of $t_{0}$ with $t_{1}=H_{1}$ and $t_{2}=H_{0}$. Assume also that, if $\Delta^{+}$is the corresponding positive system, then $\Delta^{+}\left(\mathfrak{m}_{c}\right) \subset \Delta^{+}$. Let $\widetilde{\Delta}^{+}$be the positive system obtained by substituting $-H_{0}$ with $H_{0}$ in the definition of $t_{2}$. Clearly both systems are compatible with $\mathfrak{q}$. Set $\mathfrak{m}_{c}^{+}=\sum_{\alpha \in \Delta^{+}\left(\mathfrak{m}_{c}\right)} \mathfrak{m}_{c \alpha}$.

6.7 Lemma. As $\mathfrak{m}_{c}$-modules, $\mathfrak{u}_{n 1}$ and $\mathfrak{u}_{n 2}$ are irreducible.

Proof. We need to show that $\operatorname{dim}\left(\left(\mathfrak{u}_{n i}\right)^{\mathfrak{m}_{c}^{+}}\right)=1, i=1,2$. Let $X_{\beta} \in\left(\mathfrak{u}_{n 1}\right)^{\mathfrak{m}_{c}^{+}}$ and $\eta \in \Delta^{+}\left(\mathfrak{m}_{n}\right)$; then $(\beta+\eta)\left(H_{0}\right)=2$, thus $\beta+\eta \notin \Delta$. This implies that $\left[X_{\beta}, X_{\eta}\right]=0$, hence $X_{\beta} \in \mathfrak{u}^{\mathrm{m} \cap \mathfrak{n}}$.

Since $\mathfrak{u}$ is irreducible $([\mathfrak{g}, \mathfrak{g}]$ is simple), we have that

$$
\operatorname{dim}\left(\left(\mathfrak{u}_{n i}^{\mathfrak{m}_{c}^{+}}\right)=\operatorname{dim}\left(\mathfrak{u}^{\mathfrak{m} \cap \mathfrak{n}}\right)=1,\right.
$$

as we wished to show. The proof for $u_{n 2}$ is completely analogous if we use $\widetilde{\Delta}^{+}\left(\mathfrak{m}_{n}\right)$ instead of $\Delta^{+}\left(\mathfrak{m}_{n}\right)$. 
Let

$$
\mathfrak{L}=\mathfrak{u}_{n}^{-} \oplus\left[\mathfrak{u}_{n}, \mathfrak{u}_{n}^{-}\right] \oplus \mathfrak{u}_{n} .
$$

It is easy to check using the Jacobi identity that $\mathfrak{L}$ is an ideal of $\mathfrak{l}$. Since $\mathfrak{l}$ is reductive we have that $\mathfrak{l}=\mathfrak{L} \oplus \mathfrak{l}^{0}$, where $\mathfrak{l}^{0}$ is an ideal of $\mathfrak{l}$ contained in $\mathfrak{m}_{c}$.

We can decompose $\mathfrak{l}$ further: let

$$
\begin{aligned}
& \mathfrak{l}^{1}=\mathfrak{u}_{n 1}^{-} \oplus\left[\mathfrak{u}_{n 1}, \mathfrak{u}_{n 1}^{-}\right] \oplus \mathfrak{u}_{n 1}, \\
& \mathfrak{l}^{2}=\mathfrak{u}_{n 2}^{-} \oplus\left[\mathfrak{u}_{n 2}, \mathfrak{u}_{n 2}^{-}\right] \oplus \mathfrak{u}_{n 2} .
\end{aligned}
$$

Since $\left[\mathfrak{u}_{n 1}, \mathfrak{u}_{n 2}^{-}\right]=\left[\mathfrak{u}_{n 2}, \mathfrak{u}_{n 1}^{-}\right]=0$ we see that $\mathfrak{l}^{1}, \mathfrak{l}^{2}$ are ideals in $\mathfrak{l}$ and

$$
\mathfrak{l}=\mathfrak{l}^{1} \oplus \mathfrak{l}^{2} \oplus \mathfrak{l}^{0} \text {. }
$$

Lemma 6.7 implies that the pairs $\left(\mathfrak{l}^{i}, \mathfrak{m}_{c} \cap \mathfrak{l}^{i}\right), i=1,2$, are irreducible hermitian pairs. We observe that $\Delta^{+}(\mathfrak{l})=\widetilde{\Delta}^{+}(\mathfrak{l})=\Delta^{+}\left(\mathfrak{m}_{c}\right) \cup \Delta\left(\mathfrak{u}_{n}\right)$. If $r_{i}=$ $\operatorname{rank}_{\mathbb{R}}\left(l^{i} \cap \mathfrak{l}_{0}\right)$, let $\gamma_{i 1} \leq \cdots \gamma_{i r_{i}}$ be the strongly orthogonal roots associated to $\Delta^{+}\left(\mathfrak{l}^{i}\right)$ in $\Delta\left(\mathfrak{u}_{n i}\right)$.

Observe that $\Delta\left(\mathfrak{u}_{n 1}\right)$ and $\Delta\left(\mathfrak{u}_{n 2}\right)$ are mutually strongly orthogonal roots; thus, $\left\{\gamma_{i j}\right\}$ is a set of strongly orthogonal roots. In particular, $r_{1}+r_{2} \leq r$.

6.8 Lemma. The set $\left\{\gamma_{i j}\right\}_{i=1,2 ; 1 \leq j \leq r_{i}}$ satisfies (6.1(1)).

Proof. We note that, with respect to $\Delta^{+}$, if $\alpha \in \Delta\left(\mathfrak{u}_{n 1}\right), \beta \in \Delta\left(\mathfrak{u}_{c}\right)$, and $\gamma \in$ $\Delta\left(\mathfrak{u}_{n 2}\right)$, then $\alpha \geq \beta \geq \gamma$. Therefore, if $\gamma_{1} \leq \cdots \leq \gamma_{r}$ are the corresponding strongly orthogonal roots, we have that, by construction, $\gamma_{i}=\gamma_{2 i}, 1 \leq i \leq r_{2}$. this implies that $\left\{\gamma_{2 j}\right\}$ satisfies $(6.1(1))$.

Analogously, we see that, if $\tilde{\gamma}_{1} \leq \cdots \leq \tilde{\gamma}_{r}$ are the strongly orthogonal roots associated to $\widetilde{\Delta}^{+}$, then $\tilde{\gamma}_{i}=\gamma_{1 i}, 1 \leq i \leq r_{1}$.

The obvious generalization of Proposition 6.3 applied to $\mathfrak{l}$ implies that $S\left(\mathfrak{u}_{n}^{-}\right)$ is multiplicity free as an $\mathfrak{m}_{c}$-module and the highest weights (w.r.t. $\Delta^{+}\left(\mathfrak{m}_{c}\right)$ ) are precisely those of the form:

$$
\mu=-\sum a_{i j} \gamma_{i j}, \quad a_{i 1} \geq \cdots \geq a_{i r_{i}} \geq 0, a_{i j} \in \mathbb{N}, i=1,2 .
$$

Moreover, if $v_{i j}$ is the element of $S\left(\mathfrak{u}_{n}^{-}\right)^{\mathrm{m}_{c}^{+}}$of weight $\mu_{i j}=\sum_{r=1}^{j} \gamma_{i r}$, then

$$
\mathscr{P}=\mathbb{C}\left[v_{i j}\right], \quad i=1,2, j=1, \ldots, r_{i} .
$$

If $M \in \mathbb{N}^{r_{1}} \times \mathbb{N}^{r_{2}}, M=\left(m_{11}, \ldots, m_{1 r_{1}}, m_{21}, \ldots, m_{2 r_{2}}\right)$, let

$$
v^{M}=\prod_{i, j} v_{i j}^{m_{i j}} \quad \text { and } \quad \mu(M)=\sum_{i, j} m_{i j} \mu_{i j} .
$$

By Lemma 5.5 we have that $\left(L(\Lambda)^{\mathrm{u}_{c}}\right)^{\mathrm{m}^{+} \mathrm{c}}=\mathscr{P} * 1_{\Lambda}$.

Since $\mu(M)$ is the weight of $v^{M}$ in $S\left(\mathfrak{u}_{n}^{-}\right)$, then the weight of $v^{M} * 1_{\Lambda}$ is

$$
\lambda(M)=\Lambda+\mu(M) .
$$


If $\lambda(M)+\rho_{c}$ is $\Delta(\mathfrak{k})$-regular we set

$$
w(M)=w(\lambda(M)) \in \mathscr{W}_{c}
$$

and

$$
\Delta^{+}(M)=\left\{\beta \in \Delta\left(\mathfrak{u}_{c}\right) \mid\left(\lambda(M)+\rho_{c}, \beta\right)>0\right\} .
$$

For each $i=1,2$ define $j_{i}(M)$ to be the last index such that $m_{i j} \neq 0$ and set $\mathfrak{l}(M)=j_{1}(M)+j_{2}(M)$. To $M$ we also attach a positive system $P(M)$ as follows: we write $\mu(M)=\sum_{i, j} a_{i j} \gamma_{i j}$ and fix a total order $\leq$ on the $\gamma_{i j}$ such that

$$
\begin{aligned}
& \text { (1) if } a_{i j} \geq a_{h k}, \quad \text { then } \gamma_{i j} \leq \gamma_{h k}, \\
& \text { (2) if } 1 \leq h \leq k \leq j_{i}(M) \text {, then } \gamma_{i h} \leq \gamma_{i k}, \quad i=1,2 \text {. }
\end{aligned}
$$

Such an order clearly exists since $a_{i h} \leq a_{i k}$ if $h \geq k$.

Let $\left\{\gamma_{1}, \ldots, \gamma_{r_{1}+r_{2}}\right\}$ be the set $\left\{\gamma_{i j}\right\}$ reordered according to $\leq$ (i.e., $\gamma_{i} \leq \gamma_{j}$ iff $i \leq j)$. Set $h_{i}, H_{i}, \mathfrak{t}^{+}, \mathfrak{t}^{-}$, and $B^{-}$as in the proof of 6.2. Set

$$
\Delta_{0}=\left\{\alpha \in \Delta \mid \alpha_{\mid \mathfrak{t}^{-}}=0\right\} \text {. }
$$

Clearly $\Delta_{0}$ is a root system. The choice of an ordered basis $B^{+}$of $\mathfrak{t}^{+}$ determines a positive system $\Delta_{0}^{+} \subset \Delta_{0}$. We choose $B^{+}$so that $\Delta_{0} \cap \Delta^{+}\left(\mathfrak{m}_{c}\right) \subset$ $\Delta_{0}^{+}$. Let $B=B^{+} \cup B^{-}$be the ordered basis of $i t_{0}$ defined as in 6.2. Let $P(M)$ be the positive system corresponding to this choice of $B$. The positive system $P(M)$ and the ordered set $\left\{\gamma_{i}\right\}$ satisfy the following properties.

6.11 Lemma. (1) $\Delta^{+}\left(\mathfrak{m}_{c}\right) \subset P(M)$.

(2) If $\left\{\eta_{1}, \ldots, \eta_{r}\right\}$ are the strongly orthogonal roots associated to $P(M)$, then $\gamma_{i}=\eta_{i}, \quad 1 \leq i \leq r_{1} \geq r_{2}$.

(3) $\mu(M)=-\sum_{i=1}^{\mathrm{l}(M)} a_{i}^{+} \eta_{i}=-\sum_{i=1}^{\mathrm{l}(M)} \gamma_{i}, a_{1} \geq \cdots \geq a_{\mathrm{I}(M)}>0, a_{i} \in \mathbb{N}$.

Proof. (2) follows immediately from the definition of $P(M)$ and Lemmas 6.8 and 6.2.

(3) follows from (2) and the definitions of $\mathfrak{l}(M)$ and $\left\{\gamma_{i}\right\}$.

It remains only to prove (1). Let $\alpha \in \Delta\left(\mathfrak{m}_{c}\right)$; if $\alpha_{\mid \mathfrak{t}^{-}}=0$, then $\alpha \in \Delta_{0} \cap$ $\Delta^{+}\left(\mathfrak{m}_{c}\right) \subset \Delta_{0}^{+} \subset \Delta^{+}$, so suppose that $\alpha_{\mid \mathfrak{t}^{-}} \neq 0$. This implies that $\left(\alpha, \gamma_{i j}\right) \neq 0$ for some $i, j$. By $(6.1(2))$, there is $1 \leq h \leq r_{i}$ such that $\left(\alpha, \gamma_{i h}\right)<0$ and $\left(\alpha, \gamma_{i k}\right)=0$ for $k<h$.

Since $\left(\gamma_{i h}, \gamma_{j k}\right)=0$ if $i \neq j$, it follows from $(6.1(2))$ that there is $1 \leq s \leq$ $r_{1}+r_{2}$ such that $\left(\alpha, \gamma_{s}\right)<0$ and $\left(\alpha, \gamma_{j}\right)=0$ for $j<s$, thus $\alpha\left(H_{s+1}\right)=$ $-\left(\alpha, \gamma_{s}\right) /\left(\gamma_{s}, \gamma_{s}\right)>0$ and $\alpha\left(H_{j}\right)=0$ for $j<s$. This implies that $\alpha \in \Delta^{+}$.

We now come to the main result of this section.

6.12 Theorem. $\mathscr{M}=\{\lambda(M) \mid \mathfrak{l}(M) \leq R\}$.

Proof. Let $M \in \mathbb{N}^{r_{1}} \times \mathbb{N}^{r_{2}}$ and set $\gamma_{1} \leq \cdots \leq \gamma_{r}$ as the strongly orthogonal roots corresponding to $P(M)$. According to Lemma 6.11, $\gamma_{i} \in \Delta\left(u_{n}\right)$ for $1 \leq i \leq$ 
$r_{1}+r_{2}$, and

$$
\mu(M)=-\sum_{i=1}^{\mathrm{I}(M)} a_{i} \gamma_{i}, \quad a_{1} \geq \cdots \geq a_{\mathrm{I}(M)}>0 .
$$

Let $\mathfrak{n}(M)=\sum_{\alpha \in P(M)} \mathfrak{g}_{\alpha} \cap \mathfrak{m}$, and let $v_{i}$ and $\mathfrak{g}_{i}$ be as described after Proposition 6.3. We have that there are positive integers $i_{1}, \ldots, i_{\mathrm{I}(M)}$ such that

$$
v=\prod_{S} v_{s}^{i_{s}}
$$

is the element of $S\left(\mathfrak{u}^{-}\right)^{\mathrm{n}(M)}$ of weight $\mu(M)$. Moreover, since $a_{\mathrm{I}(M)} \neq 0$, we have that $i_{\mathrm{I}(M)}>0$.

Write

$$
S\left(\mathfrak{u}^{-}\right)=S\left(\mathfrak{u}_{n}^{-}\right) \oplus \mathfrak{u}_{c}^{-} S\left(\mathfrak{u}^{-}\right)
$$

and $v=u+w$, accordingly. Let $\left\{Y_{\alpha} \mid \alpha \in \Delta\left(\mathfrak{u}^{-}\right)\right\}$be a basis of root vectors; then

$$
v=\sum_{\langle I\rangle=\mu(M)} c_{I} Y^{I} .
$$

Lemma 5.8 of [15] proves by an obvious induction that the coefficient of $\prod_{S} Y_{-\gamma_{s}}^{a_{s}}$ is nonzero in (6.15). This implies that $u \neq 0$.

Since the decomposition (6.14) is invariant under $\mathfrak{m}_{c}$ and since $\Delta^{+}\left(\mathfrak{m}_{c}\right) \subset$ $P(M)$, it follows that $u \in S\left(\mathfrak{u}_{n}^{-}\right)^{\mathrm{m}_{c}^{+}}$.

$S\left(\mathfrak{u}_{n}^{-}\right)$is multiplicity free, thus there is $c \in \mathbb{C}$ such that

$$
c u=v^{M} \text {. }
$$

Since $v \equiv u \bmod \mathfrak{u}_{c}^{-} S\left(\mathfrak{u}^{-}\right)$, it follows that

$$
v^{M} * 1_{\Lambda}=c u * 1_{\Lambda}=c p\left(v \cdot 1_{\Lambda}\right) \text {. }
$$

Theorem 6.6 implies that, if $\mathfrak{l}(M)>R$, then $v \cdot 1_{\Lambda}=0$, hence, by (6.17), $v^{M} * 1_{\Lambda}=0$ and $\lambda(M) \notin \mathscr{M}$.

If $\mathfrak{l}(M) \leq R$ we must show that $v^{M} * 1_{\Lambda} \neq 0$. Let $\mathfrak{g}^{\prime}=\mathfrak{g}_{\mathrm{l}(M)}$. By (6.4) and (6.13), $v \in S\left(\mathfrak{u}^{-} \cap \mathfrak{g}^{\prime}\right)$; thus, by (6.16), $v^{M} \in S\left(\mathfrak{u}_{n}^{-} \cap \mathfrak{g}^{\prime}\right)$. Let $\mathfrak{t}^{\prime}=\mathfrak{t} \cap \mathfrak{g}^{\prime}$ and $\Lambda_{1}^{\prime}=\Lambda_{\left.1\right|_{\mathfrak{t}^{\prime}}}, \Lambda^{\prime}=\Lambda_{\left.\right|_{\mathfrak{t}^{\prime}}}$.

Since $\langle,\rangle_{\Lambda \mid U\left(\mathfrak{g}^{\prime}\right) \cdot 1_{\Lambda} \times U\left(\mathfrak{g}^{\prime}\right) \cdot 1_{\Lambda}}$ is positive definite, it follows that

$$
U\left(\mathfrak{g}^{\prime}\right) \cdot 1_{\Lambda} \cong L\left(\mathfrak{g} \cap \mathfrak{g}^{\prime}, \Lambda\right) \text {. }
$$

The crucial observation is that

$$
U\left(\mathfrak{g}^{\prime}\right) \cdot 1_{\Lambda} \cong M\left(\mathfrak{g} \cap \mathfrak{g}^{\prime}, \Lambda^{\prime}\right) .
$$

Suppose that (6.18) is not true. Then, by (6.5) and (6.6), there are at most $\mathfrak{I}(M)$ values of $z \in\left[0, C\left(\mathfrak{g}_{0}^{1}\right) R\right]$ such that $L\left(\mathfrak{g} \cap \mathfrak{g}^{\prime},-z \Lambda_{i}^{\prime}\right)$ is $\left(\mathfrak{g}^{\prime} \cap \mathfrak{g}_{0}^{1}\right)$ unitary. Since $U\left(\mathfrak{g}^{\prime}\right) \cdot 1_{-z \Lambda_{1}}$ is unitary whenever $L\left(-z \Lambda_{1}\right)$ is, there are at least 
$R+1$ values of $z \in\left[0, C\left(\mathfrak{g}_{0}^{1}\right) R\right]$ such that $L\left(\mathfrak{g} \cap \mathfrak{g}^{\prime},-z \Lambda_{1}^{\prime}\right)$ is $\left(\mathfrak{g}^{\prime} \cap \mathfrak{g}_{0}^{1}\right)$-unitary, contradicting our assumption that $\mathfrak{l}(M) \leq R$. This proves (6.18).

Let $p^{\prime}: U\left(\mathfrak{g}^{\prime}\right) \cdot 1_{\Lambda} \rightarrow\left(U\left(\mathfrak{g}^{\prime}\right) \cdot 1_{\Lambda}\right)^{\mathfrak{u}_{c} \cap \mathfrak{g}^{\prime}}$ be the projection. By (6.18),

$$
p^{\prime}\left(v^{M} \cdot 1_{\Lambda}\right) \neq 0
$$

Suppose that $\alpha \in \Delta\left(\mathfrak{u}_{c}\right) \backslash \Delta\left(\mathfrak{u}_{c} \cap \mathfrak{g}^{\prime}\right)$, let $h_{i}=H_{\gamma_{j i}} /\left(\gamma_{i}, \gamma_{i}\right)$, and $H^{\prime}=H_{1}-$ $\sum_{j=1}^{\mathrm{l}(M)} h_{j}$. Note that $\beta\left(H^{\prime}\right) \geq 0$ for each $\beta \in \Delta(\mathfrak{u})$.

By the construction of $\mathfrak{g}^{\prime}$ (see [15]) we have that $\alpha\left(H^{\prime}\right)>0$ and $\beta\left(H^{\prime}\right)=0$ for each $\beta \in \Delta\left(\mathfrak{u} \cap \mathfrak{g}^{\prime}\right)$. In particular, $\mu(M)\left(H^{\prime}\right)=0$.

Suppose now that $X_{\alpha} \cdot p^{\prime}\left(v^{M} \cdot 1_{\Lambda}\right) \neq 0$. This implies that

$$
\lambda(M)+\alpha=\lambda-\sum_{\beta \in \Delta(\mathfrak{u})} n_{\beta} \beta \quad \text { or } \quad \alpha+\mu(M)=-\sum_{\beta \in \Delta(\mathfrak{u})} n_{\beta} \beta ;
$$

thus,

$$
0<\alpha\left(H^{\prime}\right)=(\alpha+\mu(M))\left(H^{\prime}\right)=-\sum_{\beta \in \Delta(\mathfrak{u})} n_{\beta} \beta\left(H^{\prime}\right) \leq 0 .
$$

This contradiction implies that $X_{\alpha} \cdot p^{\prime}\left(v^{M} \cdot 1_{\Lambda}\right)=0$, hence

$$
0 \neq p^{\prime}\left(v^{M} \cdot 1_{\Lambda}\right)=v^{M} * 1_{\Lambda},
$$

as we wished to show.

\section{HERMITIAN SYMMETRIC PAIRS OF CLASSICAL TYPE}

In this section we apply the results of $\S \S 5$ and 6 to the case when $[\mathfrak{g}, \mathfrak{g}]$ is of classical type. In what follows we identify $t$ and $t^{*}$ via $($,$) and adopt$ the standard " $\varepsilon_{i}$ " notation for roots and weights. Firstly we give the two main statements: the unexplained notation is as in $\S 6$ (see Tables 7.12 and 7.13 for the various data in each particular case).

7.1 Lemma. Suppose that $R \in\{0, \ldots, r-1\}$ satisfies the conditions given in Table 7.13. Then there exist $M_{0} \in \mathbb{N}^{r_{1}} \times \mathbb{N}^{r_{2}}$ and $i \in\{0, \ldots, R\}$ such that

$$
\mathscr{L}_{0}=\left\{\lambda\left(M_{0}\right)+\mu(M) \mid j_{1}(M) \leq i, j_{2}(M) \leq R-i\right\} .
$$

Furthermore, if $\alpha \in \Delta^{+}\left(M_{0}\right)$ and $\lambda\left(M_{0}\right)+\mu(M) \in \mathscr{L}_{0}$, then $(\alpha, \mu(M))=0$.

7.2 Corollary. With the hypothesis of Lemma 7.1, set $C$ and $\Lambda_{1}$ as in Table 7.12. Set $\mathbf{V}=L\left(-C R \Lambda_{1}\right)$, and let $T$ be as defined in $\S 5$. Then $\Gamma^{S-T}(\mathbf{V})$ is irreducible and unitary.

Proof. Let $\lambda=\lambda\left(M_{0}\right)+\mu(M) \in \mathscr{L}_{0}$. If we set $u_{0}=v^{M_{0}} * 1_{\Lambda}$ and $v_{\lambda}=v^{M}$, then, by 5.6 ,

$$
v_{\lambda} * u_{0}=v^{M} * v^{M_{0}} * 1_{\Lambda}=v^{M_{0}+M} * 1_{\Lambda} .
$$

Therefore, condition (ii) of Theorem 5.7 is satisfied. 
To see that condition (i) is also satisfied we observe that, since $\left[\mathfrak{u}_{n}, \mathfrak{u}_{c}\right]=$ 0 , then $(\alpha, \beta) \geq 0$ for $\alpha \in \Delta\left(\mathfrak{u}_{n}\right)$ and $\beta \in \Delta\left(\mathfrak{u}_{c}\right)$. Therefore, if $\alpha \in$ $\Delta\left(\mathfrak{u}_{c}\right) \backslash \Delta^{+}\left(M_{0}\right)$, then $(\mu(M), \alpha) \leq 0$. If $\alpha \in \Delta^{+}\left(M_{0}\right)$, then, by 7.1, $(\mu(M), \alpha)$ $=0$.

This proves that condition (i) of 5.7 is satisfied.

The preceding lemma is a simple consequence of Theorem 6.12 , but it requires a case-by-case calculation: we will give the details only for the type $C_{n}$ case; the other two cases are very similar.

Type $C_{n}$. Let the simple roots of $\mathfrak{g}$ be given as in the Dynkin diagram:

$$
\circ \alpha_{\alpha_{1}}^{\circ}-\cdots \underset{\alpha_{n-1}}{\circ} \quad \circ
$$

where $\alpha_{i}=\varepsilon_{i}-\varepsilon_{i+1}, 1 \leq i \leq n-1$, and $\alpha_{n}=2 \varepsilon_{n}$.

Let

$$
H_{0}=\frac{1}{2} \sum_{i=1}^{t} \varepsilon_{i}-\frac{1}{2} \sum_{i=t+1}^{n} \varepsilon_{i}, \quad 0<t<n,
$$

and

$$
H_{1}=\frac{1}{2} \sum_{i=1}^{n} \varepsilon_{i}
$$

If we let $\Delta^{+}$denote the positive system determined by $\Phi=\left\{\alpha_{1}, \ldots, \alpha_{n}\right\}$, then clearly $\Delta^{+}$is compatible with $\mathfrak{g}$, while $\mathfrak{m}$ is the subalgebra corresponding to:

and $\Delta(\mathfrak{u})=\left\{\varepsilon_{i}+\varepsilon_{j} \mid 1 \leq i, j \leq n\right\}$.

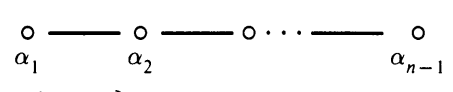

If $d=n-t$ set

so that

$$
\begin{cases}\eta_{i}=\varepsilon_{i}, & 1 \leq i \leq t, \\ \eta_{t+i}=\varepsilon_{n-i+1}, & 1 \leq i \leq d,\end{cases}
$$

$$
H_{0}=\frac{1}{2} \sum_{i=1}^{n} \eta_{i} \quad \text { and } \quad H_{1}=\frac{1}{2} \sum_{i=1}^{t} \eta_{i}-\frac{1}{2} \sum_{i=t+1}^{n} \eta_{i} \text {. }
$$

Therefore

$$
\begin{aligned}
& \mathfrak{k}: \underset{\eta_{1}-\eta_{2}}{\circ}-\circ \cdots-\underset{\eta_{n-1}-\eta_{n}}{\circ}, \\
& \Delta\left(\mathfrak{u}_{c}\right)=\left\{\eta_{i}-\eta_{j} \mid 1 \leq i \leq t<j \leq n\right\}, \\
& \mathfrak{m}_{c}: \underset{\eta_{1}-\eta_{2}}{\circ}-\circ \cdots-\underset{\eta_{t-1}-\eta_{t}}{\circ} \stackrel{\circ}{\circ} \stackrel{\circ}{\circ}-\eta_{t+1}-\cdots-\underset{\eta_{n-1}-\eta_{n}}{\circ}, \\
& \Delta\left(\mathfrak{u}_{n_{1}}\right)=\left\{\eta_{i}+\eta_{j} \mid 1 \leq i, j \leq t\right\}, \\
& \Delta\left(\mathfrak{u}_{n_{2}}\right)=\left\{-\eta_{i}-\eta_{j} \mid t+1 \leq i, j \leq n\right\} .
\end{aligned}
$$

In this setting the strongly orthogonal roots for $\mathfrak{l}$ are

$$
\gamma_{11}=2 \eta_{t}, \ldots, \gamma_{1 r_{1}}=2 \eta_{1} ; \quad \gamma_{21}=-2 \eta_{t+1}, \ldots, \gamma_{2 r_{2}}=-2 \eta_{n} ;
$$

hence, $r_{1}=t$ and $r_{2}=d$. 
If $M \in \mathbb{N}^{r_{1}} \times \mathbb{N}^{r_{2}}, i \leq t$, and $j>t$, set

$$
a_{i}=\sum_{r \geq t-i+1} m_{1 r} \text { and } b_{j}=\sum_{r \geq j-t} m_{2 r} .
$$

The obvious calculations imply that, if $i_{0}=t-j_{1}(M)+1$ and $j_{0}=t+j_{2}(M)$, then

$$
\mu(M)=-\sum_{i=i_{0}}^{t} 2 a_{i} \eta_{i}+\sum_{j=t+1}^{j_{0}} 2 b_{j} \eta_{j}
$$

and $a_{i_{0}}=m_{1 j_{1}(M)}, b_{j^{0}}=m_{2 j_{2}(M)}$. Furthermore, if $\eta_{i}-\eta_{j} \in \Delta\left(\mathfrak{u}_{c}\right)$,

$$
\left(\lambda(M)+\rho_{c}, \eta_{i}-\eta_{j}\right)=(j-i)-R-2\left(a_{i}+b_{j}\right) .
$$

7.5 Lemma. Suppose that $\lambda(M) \in \mathscr{L}$. If $\eta_{i}-\eta_{j} \in \Delta^{+}(M)$ then

$$
i<i_{0} \text { and } j>j_{0} \text {. }
$$

If $\mathfrak{l}(M)=R$, then the two conditions are equivalent.

Proof. We prove that, if $i, j$ do not satisfy (7.6) then $\eta_{i}-\eta_{j} \notin \Delta^{+}(M)$.

If is obvious from the definition that $\left(j_{0}-i_{0}\right)=\mathfrak{l}(M)-1$.

If $\lambda(M) \in \mathscr{L}$, then, by $6.12, \mathfrak{l}(M) \leq R$. If $\eta_{i}-\eta_{j} \in \Delta\left(\mathfrak{u}_{c}\right)$ and $i_{0} \leq i, j \leq$ $j_{0}$, then, by (7.4),

$\left(\lambda(M)+\rho_{c}, \eta_{i}-\eta_{j}\right)=(j-i)-R-2\left(a_{i}+b_{j}\right) \leq \mathfrak{l}(M)-1-R-2\left(a_{i}+b_{j}\right)<0$.

Suppose now that $i<i_{0}$ and $j<j_{0}$. We prove that $\eta_{i}-\eta_{j} \notin \Delta^{+}(M)$ by induction on $i_{0}-i$. If $i=i_{0}$ then

$\left(\lambda(M)+\rho_{c}, \eta_{i}-\eta_{j}\right)=(j-i)-R-2 b_{j} \leq j_{0}-i_{0}+1-R-2 b_{j}=\mathfrak{l}(M)-R-2 b_{j}<0$.

If $i<i_{0}$, then

$\left(\lambda(M)+\rho_{c}, \eta_{i-1}-\eta_{j}\right)=j-i+1-R-2 b_{j}=\left(\lambda(M)+\rho_{c}, \eta_{i}-\eta_{j}\right)+1$.

Since $\lambda(M))+\rho_{c}$ is integral, the induction hypothesis implies that

$$
\left(\lambda(M)+\rho_{c}, \eta_{i-1}-\eta_{j}\right) \leq 0 .
$$

Thus, the regularity of $\lambda(M)+\rho_{c}$ implies our claim.

A similar argument shows that, if $i_{0} \leq i$ and $j>j_{0}$, then $\eta_{i}-\eta_{j} \notin \Delta^{+}(M)$.

This proves the first part of the statement. To complete the proof, assume $\mathfrak{l}(M)=R$. Suppose that $i<i_{0}$ adn $j>j_{0}$, then

$$
\left(\lambda(M)+\rho_{c}, \eta_{i}-\eta_{j}\right)=j-i-R \geq\left(j_{0}-i_{0}\right)+2-R=1>0 .
$$

Recall that $S$ denotes $\operatorname{dim} u_{c}$.

7.7 Corollary. Suppose that $\lambda(M) \in \mathscr{L}$.

(i) If $\mathfrak{l}(M)<R$ then $l(w(M))=S$.

(ii) If $\mathfrak{l}(M)=R$ then

$$
l(w(M))=S-\left(t-j_{1}(M)\right)\left(d-j_{2}(M)\right) .
$$


Proof. Assume that $\mathfrak{l}(M)<R$. If $\eta_{i}-\eta_{j} \in \Delta^{+}(M)$, then, by 7.5, $i<i_{0}$ and $j>j_{0}$. Therefore

$$
0<\left(\lambda(M)+\rho_{c}, \eta_{i}-\eta_{j}\right)=j-i-R .
$$

Hence, $j-i>R$. Since $\mathfrak{l}(M)<R$, then $j_{0}-i_{0}=\mathfrak{l}(M)-1<R-1$. Therefore there is $i \leq r<i_{0}$ and $j_{0}<s \leq j$ such that $s-r=R$; thus,

$$
\left(\lambda(M)+\rho_{c}, \eta_{r}-\eta_{s}\right)=0,
$$

contradicting the regularity of $\lambda(M)+\rho_{c}$. This proves (i).

For (ii) observe that

$$
l(w(M))=S-\left|\Delta^{+}(M)\right|
$$

By 7.5 ,

$$
\Delta^{+}(M)=\left\{-\eta_{i}-\eta_{j} \mid 1 \leq i<i_{0}, j_{0}<j \leq n\right\}
$$

so

$$
\left|\Delta^{+}(M)\right|=\left(i_{0}-1\right)\left(n-j_{0}\right)=\left(t-j_{1}(M)\right)\left(d-j_{2}(M)\right) .
$$

Let $T_{1}=\min \left(r_{1}, R\right)$ and $f:\left\{0, \ldots, T_{1}\right\} \rightarrow \mathbb{Z}, f(j)=(t-j)(d-(R-j))$. Corollary 7.7 implies that, if $f(j) \leq 0$ for $j \in\left\{0, \ldots, T_{1}\right\}$, then, for each $\lambda(M) \in \mathscr{L}, l(w(M))=S$.

In such cases the results of [5] apply. This implies

7.8 Corollary. If $R=n-1$ then, with the notation as in 7.2, $\Gamma^{i}(\mathbf{V})=0$, $i \neq S$, and $\Gamma^{S}(\mathbf{V})$ is unitarizable w.r.t. $\mathfrak{g}_{0}$.

Proof. Since $d+t=n$, then, if $j=0, \ldots, t$,

$$
f(j)=(t-j)(d-R+j)=(t-j)(d+j-n+1) \leq 0 .
$$

Proof of Lemma 7.1 for Type $C_{n}$. Suppose that $R$ satisfies the conditions of Table 7.13. Then a straightforward calculation shows that the function $f$ has a unique maximum $i$ in $\left\{0, \ldots, T_{1}\right\}$ and that $T=f(i)>0$.

Set $r_{0}=t-i+1, s_{0}=t+R-i$, and

$$
\begin{aligned}
& m_{1}^{0}= \begin{cases}\min \left\{m \in \mathbb{N} \mid n-r_{0}-R-2 m<0\right\} & \text { if } i \neq 0, \\
0 & \text { if } i \neq 0,\end{cases} \\
& m_{2}^{0}= \begin{cases}\min \left\{m \in \mathbb{N} \mid s_{0}-1-R-2 m<0\right\} & \text { if } i \neq R, \\
0 & \text { if } i=R .\end{cases}
\end{aligned}
$$

Set $M_{0}=\left(m_{1}, \ldots, m_{n}\right)$ with $m_{i}=m_{1}^{0}, M_{R-i}=m_{2}^{0}$, and $m_{j}=0$ if $j \neq i$, $R-i$. Then

$$
\mu\left(M_{0}\right)=-\sum_{r=r_{0}}^{t} 2 m_{1}^{0} \eta_{r}+\sum_{r=t+1}^{s_{0}} 2 m_{2}^{0} \eta_{r}
$$

and one checks that $\lambda\left(M_{0}\right)+\rho_{c}$ is regular. Since $\mathfrak{l}\left(M_{0}\right)=R$ it follows from 6.12 that $\lambda\left(M_{0}\right) \in \mathscr{L}$. 
Suppose that $\lambda(M) \in \mathscr{L}_{0}$. If $\mathfrak{l}(M)<R$, then 7.7 implies that

$$
l_{0}=l(w(M))=S>S-T=l\left(w\left(M_{0}\right)\right),
$$

hence $\mathfrak{l}(M)=R$. Therefore, by 7.7,

$$
l_{0}=l(w(M))=S-f\left(j_{1}(M)\right) ;
$$

thus, $f\left(j_{1}(M)\right) \geq f(i)$. If follows that $j_{1}(M)=i, j_{2}(M)=R-i$.

A straightforward calculation shows that, by 7.5, $m_{1}^{0} \leq m_{1 i}$ and $m_{2}^{0} \leq$ $m_{2 R-i}$. It follows then, that

$$
M^{\prime}=M-M_{0} \in \mathbb{N}^{r_{1}} \times \mathbb{N}^{r_{2}}
$$

thus, $\lambda(M)=\Lambda+\mu(M)=\Lambda+\mu\left(M_{0}\right)+\mu\left(M^{\prime}\right)=\lambda\left(M_{0}\right)+\mu\left(M^{\prime}\right)$ and, since $j_{1}(M)=i$, and $j_{2}(M)=R-i$, it is clear that $j_{1}\left(M^{\prime}\right) \leq i, j_{2}\left(M^{\prime}\right) \leq R-i$. Then

$$
\mu\left(M^{\prime}\right)=-\sum_{r=r_{0}}^{t} 2 a_{r}^{\prime} \eta_{r}+\sum_{r=t+1}^{s_{0}} 2 b_{r}^{\prime} \eta_{r}
$$

with $a_{r}^{\prime} \geq 0, b_{r}^{\prime} \geq 0$. If $\eta_{r}-\eta_{s} \in \Delta^{+}\left(M_{0}\right)$, then, by 7.5, $r<r_{0}$ and $s>s_{0}$; hence, $\left(\mu\left(M^{\prime}\right), \eta_{r}-\eta_{s}\right)=0$.

It remains only to prove that, if $M^{\prime}$ satisfies the conditions above and $M=$ $M_{0}+M^{\prime}$, then $\lambda(M) \in \mathscr{L}_{0}$.

If $\alpha \in \Delta^{+}\left(M_{0}\right)$, then

$$
\left(\lambda(M)+\rho_{c}, \alpha\right)=\left(\lambda\left(M_{0}\right)+\rho_{c}, \alpha\right)+\left(\mu\left(M^{\prime}\right)+\rho_{c}, \alpha\right)=\left(\lambda\left(M_{0}\right)+\rho_{c}, \alpha\right)>0 .
$$

If $\alpha \in \Delta\left(\mathfrak{u}_{c}\right) \backslash \Delta^{+}\left(M_{0}\right)$, then $\left(\mu\left(M^{\prime}\right), \alpha\right) \leq 0$; therefore,

$$
\left(\lambda(M)+\rho_{c}, \alpha\right)=\left(\lambda\left(M_{0}\right)+\rho_{c}, \alpha\right)+\left(\mu\left(M^{\prime}\right)+\rho_{c}, \alpha\right) \leq\left(\lambda\left(M_{0}\right)+\rho_{c}, \alpha\right)<0 .
$$

This implies that $\lambda(M)+\rho_{c}$ is regular. Finally observe that

$$
\mathfrak{l}(M)=\max \left(\mathfrak{l}\left(M_{0}\right), \mathfrak{l}\left(M^{\prime}\right)\right)=R ;
$$

hence, by $6.12, \lambda(M) \in \mathscr{L}$ and, since $j_{1}(M)=j_{1}\left(M_{0}\right)=i, l(w(M))=$ $S-f(i)=l_{0}$.

It follows that $\lambda(M) \in \mathscr{L}_{0}$ and the proof is complete.

We now compute the $K$-spectrum and the lowest $K$-type of the derived functor modules considered in Corollary 7.2. set

Let $w_{0}$ be the longest element of $\mathscr{W}_{c}$ such that $w_{0}^{-1}\left(\Delta^{+}(\mathfrak{k})\right) \supseteq \Delta^{+}\left(\mathfrak{m}_{c}\right)$ and

$$
\nu_{\delta j}=w_{0}\left(\mu_{\delta j}\right), \quad \delta=1,2, j=1, \ldots, r_{\delta} .
$$

For the rest of this section we identify $K$-types with their highest weight. 
7.9 Theorem. The $K$-spectrum of $\Gamma^{S-T}(\mathbf{V})$ is multiplicity free. Moreover, there is $\lambda_{0} \in \mathrm{t}^{*}$ and $0 \leq i \leq R$ such that

(i) $\lambda_{0}$ is a lowest $K$-type for $\Gamma^{S-T}(\mathbf{V})$;

(ii) a $K$-type $\lambda$ occurs in $\Gamma^{S-T}(\mathbf{V})$ if and only if

$$
\lambda=\lambda_{0}+\sum_{j=1}^{i} m_{1 j} \nu_{1 j}+\sum_{j=1}^{R-i} m_{2 j} \nu_{2 j}, \quad m_{\delta j} \in \mathbb{N}, \delta=1,2 .
$$

Proof. We saw in $\S 5$ that

$$
\Gamma^{S-T}(\mathbf{V}) \cong \bigoplus_{\lambda \in \mathscr{L}_{0}} \Gamma^{S-T}\left(L_{c}(\lambda)\right) .
$$

Since each $\lambda \in \mathscr{L}_{0}$ occurs in $\mathbf{V}^{\mathrm{u}_{c}}$ with multiplicity one, the $K$-spectrum of $\Gamma^{S-T}(\mathbf{V})$ is multiplicity free.

It follows from (7.10), Lemma 7.1, and Corollaries 3.2 and 3.5 that a $K$-type $\lambda$ occurs in $\Gamma^{S-T}(\mathbf{V})$ if and only if

$$
\lambda=w\left(M_{0}\right)\left(\lambda\left(M_{0}\right)+\mu(\boldsymbol{M})+\rho_{c}\right)-\rho_{c} .
$$

Set $\lambda_{0}=w\left(M_{0}\right)\left(\lambda\left(M_{0}\right)+\rho_{c}\right)-\rho_{c}$. If $\lambda$ is a $K$-type occurring in $\Gamma^{S-T}(\mathbf{V})$, we must show that

$$
\left\|\lambda+2 \rho_{c}\right\| \geq\left\|\lambda_{0}+2 \rho_{c}\right\|
$$

Suppose that $\left.\lambda=w\left(M_{0}\right)\left(\lambda\left(M_{0}\right)\right)+\mu(M)+\rho_{c}\right)-\rho_{c}$ with $j_{1}(M) \leq i, j_{2}(M) \leq$ $R-i$.

Clearly $(\mu(M), \Lambda) \geq 0$ by the definition of $\Lambda$. Since $\mathfrak{u}$ is abelian, $\left(\mu(M), \mu\left(M_{0}\right)\right) \geq 0$.

We note that

$$
\rho_{c}+w\left(M_{0}\right)^{-1} \rho_{c}=2 \rho\left(\mathfrak{m}_{c}\right)+\sum_{\alpha \in \Delta^{+}\left(M_{0}\right)} \alpha
$$

hence, by 7.1 ,

$$
\begin{aligned}
\left(\mu(M), \rho_{c}+w\left(M_{0}\right)^{-1} \rho_{c}\right) & =\left(\mu(M), 2 \rho\left(\mathfrak{m}_{c}\right)\right)+\left(\mu(M), \sum_{\alpha \in \Delta^{+}\left(M_{0}\right)} \alpha\right) \\
& =\left(\mu(M), 2 \rho\left(\mathfrak{m}_{c}\right)\right) \geq 0 .
\end{aligned}
$$

Therefore,

$$
\begin{aligned}
\left\|\lambda+2 \rho_{c}\right\|^{2}= & \left\|w\left(M_{0}\right)\left(\lambda\left(M_{0}\right)+\mu(M)+\rho_{c}\right)+\rho_{c}\right\|^{2} \\
= & \left\|\left(\lambda\left(M_{0}\right)+\mu(M)+\rho_{c}\right)+w\left(M_{0}\right)^{-1} \rho_{c}\right\|^{2} \\
\geq & \left\|\lambda_{0}+2 \rho_{c}\right\|^{2}+2\left(\mu(M), \lambda\left(M_{0}\right)+\rho_{c}+w\left(M_{0}\right)^{-1} \rho_{c}\right) \\
= & \left\|\lambda_{0}+2 \rho_{c}\right\|^{2}+2(\mu(M), \Lambda)+2\left(\mu(M), \mu\left(M_{0}\right)\right) \\
& +2\left(\mu(M), \rho_{c}+w\left(M_{0}\right)^{-1} \rho_{c}\right) \\
\leq & \left\|\lambda_{0}+\rho_{c}\right\|^{2},
\end{aligned}
$$

as we wished to show. 
TABLE 7.12

\begin{tabular}{ccccc}
\hline Type & $\mathfrak{g}_{0}^{1}$ & $C=C\left(\mathfrak{g}_{0}^{1}\right)$ & $H_{1}$ & $\Lambda_{1}$ \\
\hline$A_{n}$ & $\mathfrak{u}(p, n+1-p)$ & 1 & $\frac{1}{2} \sum_{i=1}^{p} \varepsilon_{i}-\frac{1}{2} \sum_{i=p+1}^{n+1} \varepsilon_{i}$ & $H_{1}$ \\
\hline$C_{n}$ & $\mathfrak{s p}(n, \mathbb{R})$ & $\frac{1}{2}$ & $\frac{1}{2} \sum_{i=1}^{n} \varepsilon_{i}$ & $2 H_{1}$ \\
\hline$D_{n}$ & $\mathfrak{s o}^{*}(2 n)$ & 2 & $\frac{1}{2} \sum_{i=1}^{n} \varepsilon_{i}$ & $H_{1}$ \\
\hline
\end{tabular}

TABLE 7.13

\begin{tabular}{|c|c|c|c|}
\hline Type & $\mathfrak{g}_{0}$ & $H_{0}$ & admissible values for $R$ \\
\hline$A_{n}\left({ }^{*}\right)$ & $\mathfrak{u}(q, n+1-q)$ & $\begin{array}{l}\frac{1}{2} \sum_{i=1}^{t} \varepsilon_{i}-\frac{1}{2} \sum_{i=t+1}^{p} \varepsilon_{i} \\
+\frac{1}{2} \sum_{i=p+1}^{d} \varepsilon_{i}-\frac{1}{2} \sum_{i=d+1}^{n+1} \varepsilon_{i}\end{array}$ & $\begin{array}{c}R \leq r_{1}+r_{2} \text { and }, \\
\text { if } 0 \leq \frac{n+1-2(t-d)+2 R}{4} \leq r_{1}, \\
0 \leq \frac{2(t-d)-n-1+2 R}{4} \leq r_{2}, \\
n+1-2(t-d)+2 R \not \equiv 2(\bmod 4)\end{array}$ \\
\hline$C_{n}$ & $\mathfrak{s p}(n, \mathbb{R})$ & $\frac{1}{2} \sum_{i=1}^{t} \varepsilon_{i}-\frac{1}{2} \sum_{i=t+1}^{n} \varepsilon_{i}$ & $\begin{array}{c}R<n-1 \text { and } \\
\text { if } \frac{n-R}{2} \leq \min (t, n-t), \\
n-R \text { even }\end{array}$ \\
\hline$D_{n}$ & $\mathfrak{s o}^{*}(2 n)$ & $\frac{1}{2} \sum_{i=1}^{t} \varepsilon_{i}-\frac{1}{2} \sum_{i=t+1}^{n} \varepsilon_{i}$ & $\begin{array}{c}R<\left[\frac{n}{2}\right] \text { and } \\
\text { if } \frac{n-2 R}{2} \leq \min (t, n-t), \\
2 t-n+2 R \not \equiv 2(\bmod 4)\end{array}$ \\
\hline
\end{tabular}

$\left(^{*}\right)$ Here $q=t+d-p, r_{1}=\min (t, n+1-d)$, and $r_{2}=\min (d-p, p-t)$.

To complete the proof we recall that we proved that $w\left(M_{0}\right)(\mu(M))$ is $\Delta^{+}(\mathfrak{k})$ dominant. since $\left[\mathfrak{u}_{n}, \mathfrak{u}_{c}\right]=0$, it follows that $(\mu(M), \alpha) \leq 0$ for each $\alpha \in$ $\Delta\left(\mathfrak{u}_{c}\right)$; hence, $w_{0}(\mu(M))$ is $\Delta^{+}(\mathfrak{k})$-dominant.

Therefore, $w\left(M_{0}\right)(\mu(M))=w_{0}(\mu(M))$ and

$$
\begin{aligned}
\lambda & =w\left(M_{0}\right)\left(\lambda\left(M_{0}\right)+\mu(M)+\rho_{c}\right)-\rho_{c} \\
& =\lambda_{0}+w\left(M_{0}\right)(\mu(M))=\lambda_{0}+w_{0}(\mu(M)) \\
& =\lambda_{0}+\sum_{j=1}^{j_{1}(M)} m_{1 j} \nu_{2 j}+\sum_{j=1}^{j_{2}(M)} m_{2 j} \nu_{2 j} \\
& =\lambda_{0}+\sum_{j=1}^{i} m_{1 j} \nu_{2 j}+\sum_{j=1}^{R-i} m_{2 j} \nu_{2 j}
\end{aligned}
$$

since $j_{1}(M) \leq i$ and $j_{2}(M) \leq R-i$. 
TABLE 7.14. Type $A_{n}$.

Notation as in Table 7.13, $i$ as in 7.9.

$\mathfrak{k}: \underset{\eta_{1}-\eta_{2}}{\circ}-\cdots \underset{\eta_{q-1}-\eta_{q}}{\circ} \stackrel{\circ}{\circ} \stackrel{\circ}{\eta_{q+1}-\eta_{q+2}}-\cdots-\stackrel{\circ}{\eta_{n}-\eta_{n+1}}$

\begin{tabular}{lc} 
cases & $\lambda_{0}$ \\
\hline$d-p<p-t$ & $\sum_{j=1}^{q-i}\left(\frac{R}{2}-i\right) \eta_{j}+\sum_{j=q-i+i}^{q}\left(\frac{R}{2}-i+2 r_{2}-d\right) \eta_{j}$ \\
& $+\sum_{j=q+1}^{n+1-(R-i)}\left(i-\frac{R}{2}\right) \eta_{j}+\sum_{j=n+1-(R-i)+1}^{n+1}\left(i-\frac{R}{2}+2 r_{1}-t\right) \eta_{j}$ \\
\hline$d-p \geq p-t$ & $\sum_{j=1}^{q}\left(\frac{R}{2}-i\right) \eta_{j}+\sum_{j=q+1}^{q+i}\left(i-\frac{R}{2}-2 r_{2}+d\right) \eta_{j}$ \\
& $+\sum_{j=q+i+1}^{n+1-(R-i)}\left(i-\frac{R}{2}\right) \eta_{j}+\sum_{j=n+1-(R-i)+1}^{n+1}\left(i-\frac{R}{2}+2 r_{1}-t\right) \eta_{j}$
\end{tabular}

TABLE 7.15. Type $C_{n}$.

Notation as in Table 7.13.

t:

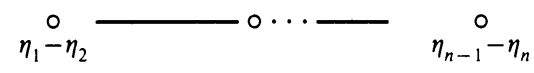

\begin{tabular}{lc} 
cases & \multicolumn{1}{c}{$\lambda_{0}$} \\
\hline$\frac{n-R}{2}>n-t, n-t$ odd & $-\sum_{j+1}^{n-R} \frac{R}{2} \eta_{j}-\sum_{i=n-R+1}^{n}\left(\frac{R}{2}+1\right) \eta_{i}$ \\
\hline$\frac{n-R}{2}>n-t, n-t$ even & $-\sum_{j=1}^{n} \frac{R}{2} \eta_{j}$ \\
\hline$\frac{n-R}{2} \leq \min (t, n-t)$, & $\sum_{j=1}^{n} \frac{n-2 t}{2} \eta_{j}$ \\
\hline$n-R$ even and $\frac{n-R}{2}$ even & $\sum_{j=1}^{R-i}\left(\frac{n-2 t}{2}+1\right) \eta_{j}+\sum_{j=R-i+1}^{n-i} \frac{n-2 t}{2} \eta_{j}$ \\
\hline$n-R$ even and $\frac{n-R}{2}$ odd & $+\sum_{j=n-i+1}^{n}\left(\frac{n-2 t}{2}-1\right) \eta_{j}$
\end{tabular}

7.11 Corollary. With the notation of 7.1 and 7.2, $\Gamma^{S-T}(\mathbf{V})$ is a highest weight module if and only if $i=0$ or $i=R$.

Proof. A $(\mathfrak{g}, K)$-module $\mathbf{M}$ is a highest weight module if and only if the set $\left\{\lambda\left(H_{0}\right) \mid \lambda\right.$ a $K$-type of $\left.\mathbf{M}\right\}$ is bounded above or below. Recall that $\mathbb{C} H_{0}$ is the center of $\mathfrak{k}$.

Note that $\nu_{\delta j}\left(H_{0}\right)=\mu_{\delta j}\left(w_{0}^{-1}\left(H_{0}\right)\right)=\mu_{\delta j}\left(H_{0}\right)=(-1)^{\delta-1} j$. (The last equality follows easily from the definition of $\mathfrak{u}_{n \delta}$.) 
TABLE 7.16. Type $D_{n}$.

Notation as in Table 7.13.

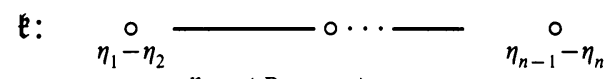

In this case we have that $\lambda_{0}=\sum_{j=1}^{n}\left(\frac{R}{2}-2 i\right) \eta_{j}$ with the values of $i$ listed below.

\begin{tabular}{cc} 
cases & $i$ \\
\hline$\frac{n-2 R}{2}>\min (t, n-t)$ & $R$ \\
\hline$\frac{n-2 R}{2} \leq \min (t, n-t)$, & $\frac{2 t-n+2 R-1}{4}$ \\
$n$ odd and $\frac{2 t-n+2 R-1}{2}$ even & \\
\hline$\frac{n-2 R}{2} \leq \min (t, n-t)$, & $\frac{2 t-n+2 R+1}{4}$ \\
$n$ odd and $\frac{2 t-n+2 R-1}{2}$ odd & \\
\hline$\frac{n-2 R}{2} \leq \min (t, n-t)$, & $\frac{2 t-n+2 R}{4}$ \\
$n$ even and $\frac{2 t-n+2 R}{2}$ even &
\end{tabular}

Suppose, for example, that $i=0$; then

$$
\begin{aligned}
& \left\{\lambda\left(H_{0}\right) \mid \lambda \text { a } K \text {-type of } \Gamma^{S-T}(\mathbf{V})\right\} \\
& \quad=\left\{\lambda\left(H_{0}\right)+\sum m_{2 j} \nu_{2 j}\left(H_{0}\right)\right\}=\left\{\lambda\left(H_{0}\right)-\sum m_{2 j} j\right\},
\end{aligned}
$$

which is clearly bounded above.

Finally, suppose that $i \neq 0$ and $i \neq R$. Then

$$
\begin{aligned}
& \left\{\lambda\left(H_{0}\right) \mid \lambda \text { a } K \text {-type of } \Gamma^{S-T}(\mathbf{V})\right\} \\
& \quad \supset\left\{\lambda\left(H_{0}\right)+m_{1} \nu_{11}\left(H_{0}\right)+m_{2} \nu_{21}\left(H_{0}\right) \mid m_{\delta} \in \mathbb{N}\right\} \\
& \quad=\left\{\lambda\left(H_{0}\right)+m_{1}-m_{2}\right\},
\end{aligned}
$$

which is not bounded.

Corollary 7.11 shows that most of the representations we constructed in 7.2 are not highest weight modules.

In the proof of Lemma 7.1 we gave an explicit procedure to compute $M_{0}$ and thus $\lambda_{0}$ in the Type $C_{n}$ case. Such a procedure applies to all cases with minor modifications: we list the result of such calculations in Tables 7.14, 7.15, and 7.16. From our expression for $\lambda_{0}$ it is also possible to compute directly the parameter $\tilde{\lambda}\left(\lambda_{0}\right)$ associated to the lowest $K$-type of a representation by Proposition 5.3.3 of [12]. The infinitesimal character of $\Gamma^{S-T}(L(\Lambda))$ is given by $\Lambda+\rho\left(\Delta^{+}\right)$, where $\Delta^{+}$is any positive system compatible with $\mathfrak{q}$.

\section{BIBLIOGRAPHY}

1. D. Barbash, The unitary dual for complex classical Lie groups, Invent. Math. 96 (1989), 103-176.

2. J. Dixmier, Algèbres enveloppantes, Cahiers Scientifique XXVII, Gauthier-Villars, Paris, 1976. 
3. T. J. Enright and N. R. Wallach, Notes on homological algebra and representations of Lie algebras, Duke Math. J. 47 (1980), 1-15.

4. T. J. Enright, R. Howe, and N. R. Wallach, A classification of unitary highest weight modules, in Representation Theory of Reductive Groups (P.C. Trombi, Ed.), Birkhäuser, Boston, Mass., 1983.

5. T. J. Enright, R. Parthasarathy, N. R. Wallach, and J. A. Wolf, Unitary derived functor modules with small spectrum, Acta Math. 154 (1985), 105-243.

6. Harish-Chandra, Representations of semisimple Lie groups. I, Trans. Amer. Math. Soc. 75 (1953), 185-243.

7. __ Representations of semisimple Lie groups. VI, Amer. J. Math. 78 (1956), 564-628.

8. S. Helgason, Differential geometry and symmetric spaces, Academic Press, New York, 1962.

9. J. C. Jantzen, Kontravariante Formen auf Induzierten Darstellungen Halbeinfacher LieAlgebren, Math. Ann. 226 (1977), 53-65.

10. __, Moduln mit einem Höchsten Gewicht, Lecture Notes in Math., vol. 750, SpringerVerlag, New York, 1979.

11. W. Schmid, Die Randwerte Holomorpher Funktionen auf Hermitesch Symmetrischen Räumen, Invent. Math. 9 (1969/70), 61-80.

12. D. A. Vogan, Jr., Representations of real reductive Lie groups, Birkhäuser, Boston, Mass., 1981.

13. _ The unitary dual of $\mathbf{G L}(n)$ over an archimedean field, Invent. Math. 83 (1986), 449-505.

14. _ Unitarizability of certain series of representations, Ann. of Math. (2) 120 (1984), 141-187.

15. N. R. Wallach, The analytic continuation of the discrete series. II, Trans. Amer. Math. Soc. 251 (1979), 19-37.

16. __ On the unitarizability of derived functor modules, Invent. Math. 78 (1984), 131-141.

17. __, Real reductive groups. I, Academic Press, 1988.

18. G. J. Zuckerman, Tensor products of finite and infinite dimensional representations of semisimple Lie groups, Ann. of Math. (2) 106 (1977), 295-308.

Department of Mathematics, University of Trento, Trento, Italy 